\title{
ENTROPY AND FLATNESS IN LOCAL ALGEBRAIC DYNAMICS
}

\author{
Mahdi Majidi-Zolbanin, Nikita Miasnikov, and Lucien Szpiro
}

\begin{abstract}
For a local endomorphism of a noetherian local ring we introduce a notion of entropy, along with two other asymptotic invariants. We use this notion of entropy to extend numerical conditions in Kunz' regularity criterion to every contracting endomorphism of a noetherian local ring, and to give a characteristic-free interpretation of the definition of Hilbert-Kunz multiplicity. We also show that every finite endomorphism of a complete noetherian local ring of equal characteristic can be lifted to a finite endomorphism of a complete regular local ring. The local ring of an algebraic or analytic variety at a point fixed by a finite self-morphism inherits a local endomorphism whose entropy is well-defined. This situation arises at the vertex of the affine cone over a projective variety with a polarized self-morphism, where we compare entropy with degree.
\end{abstract}

2010 Mathematics Subject Classification: 37P05, 13D40, 37P55, 14B25, 37P99.

Key words: Local algebraic dynamics, local entropy, endomorphism of finite length, Kunz' regularity criterion, generalized Hilbert-Kunz multiplicity.

\section{Contents}

Index of notations $\quad 510$

0 . Introduction $\quad 510$

1. Local entropy 515

1.1. Examples of rings with endomorphisms 515

1.2. Existence and estimates for local entropy 516

1.3. Properties of local entropy $\quad 520$

1.4. Reduction to equal characteristic 523

1.5. Local entropy and degree 524

1.6. Entropy at the vertex of the cone over a projective variety 526

1.7. Integral endomorphisms 528

2. Regularity, flatness and entropy 530

2.1. Kunz' regularity criterion via local entropy 531

The second and third authors received funding from the NSF Grants DMS-0854746 and DMS-0739346. 
2.2. Generalized Hilbert-Kunz multiplicity

2.3. Endomorphisms of complete equicharacteristic local rings 536

\section{Index of notations}

Unless otherwise stated, all rings in this paper are assumed to be noetherian, local, commutative and with identity element 1 . The following notation is used:

\begin{tabular}{|c|c|}
\hline $\begin{array}{l}\varphi^{n} \\
\operatorname{length}_{R}(M)\end{array}$ & $\begin{array}{l}n \text {-fold composition of } \varphi \text { with itself for a self-map } \varphi: X \rightarrow X \text { of a space. } \\
\text { length of an } R \text {-module of finite length } M\end{array}$ \\
\hline length $(f)$ & $\operatorname{length}_{S}(S / f(\mathfrak{m}) S)$ of the closed fiber of a local homomorphism $f:(R, \mathfrak{m}) \rightarrow S$. \\
\hline$h_{\mathrm{loc}}(\varphi)$ & local entropy of an endomorphism of finite length $\varphi: R \rightarrow R$ \\
\hline$q_{\varphi}$ & $\exp \left(h_{\mathrm{loc}}(\varphi) / d\right)$ with $d=\operatorname{dim} R, \varphi: R \rightarrow R$ an endomorphism of finite length. \\
\hline$f_{*} N$ & $R$-module obtained from an $S$-module $N$ via a homomorphism $f: R \rightarrow S$ \\
\hline$f_{*} S$ & $R$-algebra obtained from a ring $S$ via a homomorphism $f: R \rightarrow S$ (see [8]). \\
\hline $\operatorname{deg}(f)$ & the rank of $f_{*} S$ where $f: R \rightarrow S$ is a finite homomorphism and $R$ is a domain. \\
\hline$\mu_{R}(M)$ & minimum number of generators of a finitely generated $R$-module $M$. \\
\hline$R$ & set of all minimal prime ideals of a ring $R$. \\
\hline & c $R$ corresponding to a ho \\
\hline
\end{tabular}

\section{Introduction}

Let $\varphi: X \rightarrow X$ be a self-morphism of an algebraic or analytic variety $X$ and assume that $P \in X$ is fixed by $\varphi$. If $\varphi$ is finite, or if $P$ is totally ramified, then the local ring $\mathcal{O}_{X, P}$ inherits a local endomorphism $\varphi_{P}^{\sharp}$ whose closed fiber is an artinian ring. This situation arises, for example, at the vertex of the affine cone over a projective variety with a polarized self-morphism, or at infinity for a polynomial map of the projective line $\mathbb{P}^{1}$. There is a recent literature on constructing examples of finite endomorphisms $\varphi_{P}^{\sharp}: \mathcal{O}_{X, P} \rightarrow \mathcal{O}_{X, P}$ that are not automorphisms, with $(X, P)$ a normal singularity. References include $[\mathbf{6}, 6.2-6.3]$ and $[\mathbf{1 6}$, 2.3-2.5]. Other authors, see e.g. [11], have addressed the question of whether the existence of a finite surjective self-morphism of degree $>1$ of a normal variety imposes restrictions on its local geometry or the nature of its singularities.

In [17] Favre and Jonsson studied a number of local invariants of endomorphisms of local rings of germs of analytic functions over the complex numbers (also see $[\mathbf{2 0}]$ ). Their study of these local invariants explains the obstructions encountered in establishing an equidistribution theorem 
for $(1,1)$-currents, that extends results of Brolin [10], Lyubich [32], the third author, Ullmo and Zhang [48]. The work of Favre and Jonsson has also been a key to many subsequent works, including [49], [14], and [39].

This paper will primarily be concerned with dynamical systems generated by endomorphisms of finite length of noetherian local rings.

Definition 1. A local homomorphism $f:(R, \mathfrak{m}) \rightarrow(S, \mathfrak{n})$ of noetherian local rings is of finite length, if one of the following equivalent conditions holds:

a) $f(\mathfrak{m}) S$ is $\mathfrak{n}$-primary.

b) If $\mathfrak{p}$ is a prime ideal of $S$ such that $f^{-1}(\mathfrak{p})=\mathfrak{m}$, then $\mathfrak{p}=\mathfrak{n}$.

c) If $\mathfrak{q}$ is any $\mathfrak{m}$-primary ideal of $R$, then $f(\mathfrak{q}) S$ is $\mathfrak{n}$-primary.

Note that for a homomorphism of noetherian local rings, finite $\Rightarrow$ integral $\Rightarrow$ finite length and finite $\Rightarrow$ quasi-finite $\Rightarrow$ finite length. Also the composition of two homomorphisms of finite length is again of finite length.

Definition 2. A local endomorphism $f:(R, \mathfrak{m}) \rightarrow(R, \mathfrak{m})$ of a noetherian local ring $R$ is called contracting if for every $x \in \mathfrak{m}$ the sequence $\left\{f^{n}(x)\right\}_{n \geqslant 1}$ converges to 0 in the $\mathfrak{m}$-adic topology of $R$.

Remark 3. The terminology of contracting endomorphism is due to Avramov, Hochster, Iyengar, Miller, and Yao, see [3, p. 80] or [2, p. 2]. This terminology is due to the fact that such endomorphisms are contracting in the $\mathfrak{m}$-adic topology of the ring. However, if $R$ is a local ring admitting a contracting endomorphism in the sense of Definition 2, then the closed point of $\operatorname{Spec} R$ corresponds to a super-attracting fixed point in the sense of complex (or non-archimedean) dynamics. In [22] for instance, an endomorphism satisfying the condition of Definition 2 is called super-attracting.

Lemma 4. Let $(R, \mathfrak{m})$ be a noetherian local ring of embedding dimension $\delta$. A local endomorphism $\varphi: R \rightarrow R$ is contracting if and only if $\varphi^{\delta}(\mathfrak{m}) \subset \mathfrak{m}^{2}$.

Proof: See [3, Lemma 12.1.4].

Definition 5. A local algebraic dynamical system $(R, \varphi)$ is a noetherian local ring $R$ together with an endomorphism of finite length $\varphi: R \rightarrow R$. A morphism $f:(R, \varphi) \rightarrow(S, \psi)$ between two local algebraic dynamical systems is a local homomorphism $f: R \rightarrow S$ such that $\psi \circ f=f \circ \varphi$.

In the first part of this paper three asymptotic invariants are introduced for an endomorphism $\varphi$ of finite length of a noetherian local 
$\operatorname{ring}(R, \mathfrak{m})$ :

$$
\begin{aligned}
h_{\mathrm{loc}}(\varphi) & =\lim _{n \rightarrow \infty} \frac{1}{n} \log \left(\operatorname{length}_{R}\left(R / \varphi^{n}(\mathfrak{m}) R\right)\right) \\
v_{h}(\varphi) & =\lim _{n \rightarrow \infty} \frac{1}{n} \log \left(\max \left\{r \mid \varphi^{n}(\mathfrak{m}) \subseteq \mathfrak{m}^{r}\right\}\right) \\
w_{h}(\varphi) & =\lim _{n \rightarrow \infty} \frac{1}{n} \log \left(\min \left\{r \mid \mathfrak{m}^{r} \subseteq \varphi^{n}(\mathfrak{m})\right\}\right) .
\end{aligned}
$$

Our first theorem asserts:

Theorem 1. Let $(R, \mathfrak{m}, \varphi)$ be a local algebraic dynamical system. Suppose $R$ is of dimension $d$ and embedding dimension $\delta$. Then

a) The invariants $h_{\mathrm{loc}}(\varphi), v_{h}(\varphi)$ and $w_{h}(\varphi)$ are finite and non-negative.

b) $0 \leqslant d \cdot v_{h}(\varphi) \leqslant h_{\mathrm{loc}}(\varphi) \leqslant d \cdot w_{h}(\varphi)$.

c) If $\varphi$ is in addition contracting, then $\delta \cdot h_{\mathrm{loc}}(\varphi) \geqslant d \cdot \log 2$.

d) If $R$ is of prime characteristic $p>0$, then $h_{\mathrm{loc}}\left(\right.$ Frobenius $\left._{R}\right)=d$. $\log p$.

We call the main invariant $h_{\mathrm{loc}}(\varphi)$ local entropy. Roughly speaking, in the theory of dynamical systems, entropy is a notion that measures the rate of increase in dynamical complexity as the system evolves with time [51, p. 313]. Various forms of entropy exist in the literature. For instance, Adler, Konheim, and McAndrew introduced the notion of topological entropy in [1] for continuous maps of compact topological spaces. Measure-theoretic entropy was introduced by Kolmogorov in [28] and later improved by Sină in [47] for measure-preserving morphisms of probability spaces, and in [4] Bellon and Viallet introduced a notion of algebraic entropy for dominant rational self-maps of projective space.

We show that our notion of local entropy shares many standard properties of topological entropy. For instance, writing $h(\varphi)$ for entropy of a self-map $\varphi$ of a space $X$, both local and topological entropies satisfy:

1) $h\left(\varphi^{t}\right)=t \cdot h(\varphi)$ for all $t \in \mathbb{N}$, where $\varphi^{t}=\varphi \circ \varphi \circ \cdots \circ \varphi(t$ copies $)$.

2) If $Y \subset X$ is a closed $\varphi$-stable subspace, then $h\left(\varphi \uparrow_{Y}\right) \leqslant h(\varphi)$.

3) If $f: X \rightarrow X^{\prime}$ is an isomorphism, then $h(\varphi)=h\left(f \circ \varphi \circ f^{-1}\right)$.

4) If $X=\bigcup Y_{i}, i=1, \ldots, m$, where the $Y_{i}$ are closed $\varphi$-stable subspaces, then $h(\varphi)=\max \left\{h\left(\left.\varphi\right|_{Y_{i}}\right): 1 \leqslant i \leqslant m\right\}$.

The various invariants that measure the complexity of a given system are often related (see, e.g., [33]). We compare $h_{\mathrm{loc}}(\varphi)$ to degree of $\varphi$ when $R$ is a domain and $\varphi: R \rightarrow R$ is finite. Particular attention is given to the local ring of the vertex of the affine cone of an integral projective 
variety equipped with a polarized self-morphism, for which we prove (see Proposition 40) an analogue of a result of Gromov in $[\mathbf{2 3}]$, where he showed topological entropy of a self-morphism of $\mathbb{P}^{n}(\mathbb{C})$ is equal to logarithm of its topological degree.

The invariant $v_{h}(\varphi)$ has been studied by Favre and Jonsson in [19] and $[\mathbf{1 7}]$ in a different guise. In $[\mathbf{1 9}$, Theorem A] they prove the remarkable result that if $k$ is an arbitrary field and $\varphi$ is a $k$-endomorphism of the ring $k \llbracket X, Y \rrbracket$, then $v_{h}(\varphi)$ is the logarithm of a quadratic algebraic integer. A priori it is assumed in [19, Theorem A] that the characteristic of the field is equal to 0 , but the method relies on the technique of key polynomials of [18, Appendix E], which is valid in arbitrary characteristic. Bellon and Viallet have conjectured in [4] that their notion of algebraic entropy for dominant rational self-maps of projective space is also always the logarithm of an algebraic integer (see also [46, Conjecture 8]). This conjecture is proved for monomial self-maps in [24, Corollary 6.4]. It is, therefore natural to ask a similar question about the invariants $h_{\mathrm{loc}}(\varphi), v_{h}(\varphi)$ and $w_{h}(\varphi)$ :

Question 6. Let $(R, \varphi)$ be a local algebraic dynamical system. Are the invariants $h_{\mathrm{loc}}(\varphi), v_{h}(\varphi)$ and $w_{h}(\varphi)$ always logarithms of algebraic integers?

An endomorphism $\varphi: R \rightarrow R$ induces a self-morphism ${ }^{a} \varphi$ of Spec $R$. When $\varphi$ is integral and $\operatorname{Spec} R=V(\operatorname{ker} \varphi)$, we show that ${ }^{a} \varphi$ permutes the irreducible components of $\operatorname{Spec} R$. As a result, irreducible components of $\operatorname{Spec} R$ are stable under an iteration of $\varphi$. Even when $\operatorname{Spec} R \neq V(\operatorname{ker} \varphi)$, there exist irreducible components that are stable under an iteration of $\varphi$.

In the second part of this paper we study the case of regular local rings. We use local entropy to extend numerical conditions of Kunz' regularity criterion to arbitrary contracting endomorphisms of finite length. This result of Kunz was established as a converse to a result of Peskine and the third author [40, Theorem 1.7], stating that the pullback of a free resolution of a module of finite projective dimension by the Frobenius endomorphism is a free resolution of the pullback of the module. This statement is still mysterious for a general endomorphism of a local ring, even for its iterates. One should note, however, that it is true for resolutions of modules of finite length. (Apply the acyclicity lemma [40, Lemma 1.8].)

The second theorem of this paper asserts:

Theorem 2. Let $(R, \mathfrak{m}, \varphi)$ be a local algebraic dynamical system and let $d$ be the dimension of $R$. Let $h_{\mathrm{loc}}(\varphi)$ be the local entropy of this system and define $q_{\varphi}:=\exp \left(h_{\mathrm{loc}}(\varphi) / d\right)$. Consider the following conditions: 
a) $R$ is regular.

b) $\varphi: R \rightarrow R$ is flat.

c) length $(R / \varphi(\mathfrak{m}) R)=q_{\varphi}^{d}$.

d) length $\left(R / \varphi^{n}(\mathfrak{m}) R\right)=q_{\varphi}^{\text {nd }}$ for some $n \in \mathbb{N}$.

Then $a) \Rightarrow b) \Rightarrow c) \Rightarrow d$ ). If in addition $\varphi$ is contracting, all these conditions are equivalent.

Avramov, Iyengar and Miller have proved the equivalence of conditions a) and b) (and more results) in [3] using different methods. We use Herzog's proof from [25, Satz 3.1] that is based on a nontrivial argument in local cohomology, to prove the implication b) $\Rightarrow$ a). He originally wrote it for the Frobenius endomorphism. This part of our proof has been previously used by Bruns and Gubeladze in [12, Lemma 3]. Our proof of the implication d) $\Rightarrow$ b) utilizes a flatness criterion that is due to Nagata.

We propose a characteristic-free interpretation of definition of the Hilbert-Kunz multiplicity, see [37], in terms of local entropy. From Theorem 2 it quickly follows that the Hilbert-Kunz multiplicity of a regular local ring with respect to any endomorphism of finite length is 1 . This is a well-known fact in the case of the Frobenius endomorphism. The existence of the Hilbert-Kunz multiplicity attached to an arbitrary endomorphism of finite length, however, remains an open question.

Our third theorem in this paper is inspired by results of Fakhruddin [15, Corollary 2.2], and Bhatnagar and the third author [5, Theorem 2.1] on extending a polarized self-morphism of a projective variety over an infinite field to an ambient projective space. Recently in [41] Poonen gave a proof for the main result of [5] over finite fields. Here we consider a similar lifting problem for an endomorphism of finite length of an equicharacteristic complete noetherian local ring, and prove a CohenFakhruddin type structure theorem. Note that it is easy to see that if a local ring has a nonzero contracting endomorphism, it must be of equal characteristic. In our local version of Fakhruddin's result we do not assume our fields to be infinite.

Our third theorem asserts:

Theorem 3. Let $(A, \varphi)$ be a local algebraic dynamical system and assume that $A$ is a homomorphic image $\pi: R \rightarrow A$ of an equicharacteristic complete regular local ring $R$. Then $\varphi$ can be lifted (non uniquely) to an endomorphism of finite length $\psi$ of $R$ such that $\pi \circ \psi=\varphi \circ \pi$. Thus, $\pi:(R, \psi) \rightarrow(A, \varphi)$ becomes a morphism of local algebraic dynamical systems. 
The converse problem of characterizing homomorphic images of a regular local ring $R$ to which a given endomorphism of finite length $\psi$ of $R$ descends, is an open question. Equivalently, this question is asking for characterization of ideals $\mathfrak{a} \subset R$ for which $\psi(\mathfrak{a}) \subseteq \mathfrak{a}$. If $\mathfrak{a}$ is such an ideal then $\psi$ descends to give an endomorphism of finite length of $R / \mathfrak{a}$.

The results and questions that we discussed in this introduction, as well as the equidistribution results for the measure of maximal entropy obtained by Brolin [10], Lyubich [32], the third author, Ullmo and Zhang [48], have motivated us to study local and global invariants of schemes with self-morphisms.

\section{Local entropy}

\subsection{Examples of rings with endomorphisms.}

Example 7. If $R$ is a noetherian local ring of positive prime characteristic $p$, then the Frobenius endomorphism $x \mapsto x^{p}$ is contracting and of finite length.

Example 8. A power series ring $R:=k \llbracket X_{1}, \ldots, X_{n} \rrbracket$ over a field $k$ has many endomorphisms of finite length. If elements $f_{1}, \ldots, f_{n}$ of $R$ generate an ideal of height $n$ in $R$, then we obtain an endomorphism of finite length by setting $X_{i} \mapsto f_{i}$ for $1 \leqslant i \leqslant n$. Conversely, in Theorem 3 we will show using Cohen's structure theorem that every endomorphism of finite length of a complete equicharacteristic local ring is induced by an endomorphism of a power series ring.

Definition 9. Let $\varphi: R \rightarrow R$ be an endomorphism of a noetherian local ring. An ideal $\mathfrak{a}$ of $R$ is called $\varphi$-stable, if $\varphi(\mathfrak{a}) R \subseteq \mathfrak{a}$.

Example 10. Let $R:=k \llbracket X_{1}, \ldots, X_{n} \rrbracket$ be a power series ring over a field $k$, and let $\varphi$ be an endomorphism of finite length of $R$, e.g., as defined in Example 8. Let $z \neq 0$ be an arbitrary element of the maximal ideal of $R$. Then the ideal a generated by $z, \varphi(z), \varphi^{2}(z), \ldots$ (orbit of $z$ under $\varphi$ ) is $\varphi$-stable. Thus $\varphi$ induces an endomorphism of finite length on $R / \mathfrak{a}$. Moreover, if $\varphi$ is contracting, then so is the induced map. Macaulay 2 can be used to generate examples of this type. We mention three examples here. In these examples $k$ is a field of characteristic zero, $R$ and $\mathfrak{a}$ are as above and $\mu(\cdot)$ denotes the minimum number of generators of a finitely generated $R$-module.

a) $n=4, z=X_{2}^{2}+X_{3}^{5}$. Define $\varphi$ as $X_{1} \mapsto X_{1}^{3}, X_{i} \mapsto X_{i}^{2}$ for $i=2,3$ and $X_{4} \mapsto X_{4}^{7}$. Then $\mu(\mathfrak{a})=2$ and $\operatorname{dim} R / \mathfrak{a}=2$. 
b) $n=7, z=X_{1} X_{2} X_{3}+X_{4}^{3}+X_{5}^{2} X_{6}+X_{7}^{3}$. Define $\varphi$ as $X_{i} \mapsto X_{i}^{2}$, for $2 \leqslant i \leqslant 6$ and $X_{1} \mapsto X_{7}^{2}, X_{7} \mapsto X_{1}^{2}$. Then $\mu(\mathfrak{a})=5$ and $\operatorname{dim} R / \mathfrak{a}=3$.

c) $n=8, z=X_{1} X_{4}^{5} X_{8}^{2}+X_{3} X_{5}^{4}+X_{2} X_{6}^{3}+X_{7}$. Define $\varphi$ as $X_{i} \mapsto X_{i}^{2}$, for $3 \leqslant i \leqslant 8$ and $X_{1} \mapsto X_{2}^{2}, X_{2} \mapsto X_{1}^{2}$. Then $\mu(\mathfrak{a})=5$ and $\operatorname{dim} R / \mathfrak{a}=4$.

Example 11. Let $R:=k \llbracket X_{1}, \ldots, X_{n} \rrbracket$ be a power series ring over a field $k$, and let $\mathfrak{a}$ be an ideal of $R$ with generators that can be expressed in the form monomial $=$ monomial. Then the endomorphism of $R$ given by $X_{i} \mapsto X_{i}^{r}$ for an integer $r>1$, induces a contracting endomorphism of finite length on $R / \mathfrak{a}$. In more geometric terms this example says any singularity of a toric variety admits a contracting self-morphism.

1.2. Existence and estimates for local entropy. In this section we prove Theorem 1 . We will need a lemma that is often used in dynamical systems. For a proof we refer to [50, Theorem 4.9].

Lemma (Fekete). Let $\left\{a_{n}\right\}_{n \geqslant 1}$ be a sub-additive (super-additive) sequence of real numbers, that is, $a_{n+m} \leqslant a_{n}+a_{m}$ (resp. $a_{n+m} \geqslant a_{n}+$ $\left.a_{m}\right)$ for all $n, m \in \mathbb{N}$. Then $\lim _{n \rightarrow \infty} a_{n} / n$ exists and equals $\inf _{n}\left\{a_{n} / n\right\}$ (resp. $\left.\sup _{n}\left\{a_{n} / n\right\}\right)$. (The limit could be $-\infty$ (resp. $\left.\infty\right)$ but if $a_{n} \geqslant c \cdot n$ (resp. $a_{n} \leqslant c \cdot n$ ) for a number $c>0$ and all $n \in \mathbb{N}$, then the limit will be finite.)

We will also need the proposition that follows:

Proposition 12. Let $f:(R, \mathfrak{m}) \rightarrow S$ be a homomorphism of finite length of noetherian local rings. Let $M$ be an $R$-module of finite length. Then

a) $M \otimes_{R} S$ is of finite length as an $S$-module.

b) length $_{S}\left(M \otimes_{R} S\right) \leqslant \operatorname{length}_{S}(S / f(\mathfrak{m}) S) \cdot \operatorname{length}_{R}(M)$.

c) If $f$ is flat, then length ${ }_{S}\left(M \otimes_{R} S\right)=\operatorname{length}_{S}(S / f(\mathfrak{m}) S) \cdot \operatorname{length}_{R}(M)$.

Proof: By induction on length ${ }_{R}(M)$.

Definition 13. Let $f:(R, \mathfrak{m}) \rightarrow(S, \mathfrak{n})$ be a local homomorphism of finite length of noetherian local rings. We define

$$
\begin{aligned}
\operatorname{length}(f) & =\operatorname{length}(S / f(\mathfrak{m}) S) \\
v(f) & =\max \left\{r \mid f(\mathfrak{m}) S \subset \mathfrak{n}^{r}\right\} \\
w(f) & =\min \left\{r \mid \mathfrak{n}^{r} \subset f(\mathfrak{m}) S\right\} .
\end{aligned}
$$

Remark 14. The definition of $v(f)$ and $w(f)$ was inspired by similar definitions of Samuel in [43, p. 11]. Note that $\mathfrak{n}^{w(f)} \subset f(\mathfrak{m}) S \subset \mathfrak{n}^{v(f)}$. Thus: $v(f) \leqslant w(f)$. 
Lemma 15. Let $f:(R, \mathfrak{m}) \rightarrow(S, \mathfrak{n})$ and $g:(S, \mathfrak{n}) \rightarrow(T, \mathfrak{p})$ be two homomorphisms of finite length of noetherian local rings. Write length $(f)$, length $(g)$, length $(g \circ f)$ for the lengths of the closed fibers of $f, g$ and $g \circ f$, respectively. Then

a) $\operatorname{length}(g) \leqslant \operatorname{length}(g \circ f) \leqslant \operatorname{length}(g) \cdot \operatorname{length}(f)$.

b) If $g$ is flat then length $(g \circ f)=\operatorname{length}(g) \cdot \operatorname{length}(f)$.

c) $v(g \circ f) \geqslant v(g) \cdot v(f)$.

d) $w(g \circ f) \leqslant w(g) \cdot w(f)$.

Proof: For a) and b) apply Proposition 12, using the canonical isomorphism of $T$-modules $(S / f(\mathfrak{m}) S) \otimes_{S} T \cong T / g(f(\mathfrak{m}) S) T$ (see, e.g., $[\mathbf{7}$, Chapter II, §3.6, Corollaries 2 and 3, pp. 253-254]). For c) and d) using the fact that for an ideal $\mathfrak{a}$ of $S$ and $n \in \mathbb{N}, g\left(\mathfrak{a}^{n}\right) T=(g(\mathfrak{a}) T)^{n}$, we can write

$$
((g \circ f)(\mathfrak{m})) T=g(f(\mathfrak{m}) S) T \subset g\left(\mathfrak{n}^{v(f)}\right) T=(g(\mathfrak{n}) T)^{v(f)} R \subset \mathfrak{p}^{v(g) v(f)},
$$

and

$$
\mathfrak{p}^{w(g) w(f)} \subset(g(\mathfrak{n}) T)^{w(f)}=g\left(\mathfrak{n}^{w(f)}\right) T \subset g(f(\mathfrak{m}) S) T=((g \circ f)(\mathfrak{m})) T .
$$

By definition of $v(g \circ f)$ and $w(g \circ f)$ then we obtain $v(g \circ f) \geqslant v(g) \cdot v(f)$ and $w(g \circ f) \leqslant w(g) \cdot w(f)$, respectively.

Theorem 1. Let $(R, \mathfrak{m}, \varphi)$ be a local algebraic dynamical system. Suppose $R$ is of dimension $d$ and embedding dimension $\delta$. Define

$$
\begin{aligned}
h_{\mathrm{loc}}(\varphi) & :=\lim _{n \rightarrow \infty} \frac{1}{n} \log \operatorname{length}\left(\varphi^{n}\right)=\lim _{n \rightarrow \infty} \frac{1}{n} \log \left(\operatorname{length}_{R}\left(R / \varphi^{n}(\mathfrak{m}) R\right)\right) \\
v_{h}(\varphi) & :=\lim _{n \rightarrow \infty} \frac{1}{n} \log v\left(\varphi^{n}\right)=\lim _{n \rightarrow \infty} \frac{1}{n} \log \left(\max \left\{r \mid \varphi^{n}(\mathfrak{m}) \subseteq \mathfrak{m}^{r}\right\}\right) \\
w_{h}(\varphi) & :=\lim _{n \rightarrow \infty} \frac{1}{n} \log w\left(\varphi^{n}\right)=\lim _{n \rightarrow \infty} \frac{1}{n} \log \left(\min \left\{r \mid \mathfrak{m}^{r} \subseteq \varphi^{n}(\mathfrak{m})\right\}\right) .
\end{aligned}
$$

a) The invariants $h_{\mathrm{loc}}(\varphi), v_{h}(\varphi)$ and $w_{h}(\varphi)$ are finite and non-negative. In addition, $h_{\operatorname{loc}}(\varphi)=\inf _{n}\left\{\left(\log \operatorname{length}\left(\varphi^{n}\right)\right) / n\right\}, v_{h}(\varphi)=$ $\sup _{n}\left\{\left(\log v\left(\varphi^{n}\right)\right) / n\right\}$ and $w_{h}(\varphi)=\inf _{n}\left\{\left(\log w\left(\varphi^{n}\right)\right) / n\right\}$.

b) $0 \leqslant d \cdot v_{h}(\varphi) \leqslant h_{\mathrm{loc}}(\varphi) \leqslant d \cdot w_{h}(\varphi)$.

c) If $\varphi$ is in addition contracting, then $\delta \cdot h_{\mathrm{loc}}(\varphi) \geqslant d \cdot \log 2$.

d) If $R$ is of prime characteristic $p>0$, then $h_{\mathrm{loc}}\left(\right.$ Frobenius $\left._{R}\right)=d$. $\log p$.

We call the invariant $h_{\mathrm{loc}}(\varphi)$ local entropy of $\varphi$. 
Proof: a) Apply Fekete's Lemma, taking $a_{n}$ to be $\log \operatorname{length}\left(\varphi^{n}\right), \log w\left(\varphi^{n}\right)$ and $\log v\left(\varphi^{n}\right)$, respectively. The sub-additivity of $\left\{\log \operatorname{length}\left(\varphi^{n}\right)\right\}$ and $\left\{\log w\left(\varphi^{n}\right)\right\}$ and super-additivity of $\left\{\log v\left(\varphi^{n}\right)\right\}$ were established in Lemma 15. By Lemma 15 and Remark 14 , for every $n \in \mathbb{N}$

$$
1 \leqslant[v(\varphi)]^{n} \leqslant v\left(\varphi^{n}\right) \leqslant w\left(\varphi^{n}\right) \leqslant[w(\varphi)]^{n} .
$$

These inequalities give the finiteness and non-negativity of the limits.

b) From Definition 13 we get $\mathfrak{m}^{w\left(\varphi^{n}\right)} \subset \varphi^{n}(\mathfrak{m}) R \subset \mathfrak{m}^{v\left(\varphi^{n}\right)}$. Thus

$$
\operatorname{length}_{R}\left(R / \mathfrak{m}^{v\left(\varphi^{n}\right)}\right) \leqslant \operatorname{length}\left(R / \varphi^{n}(\mathfrak{m}) R\right) \leqslant \operatorname{length}_{R}\left(R / \mathfrak{m}^{w\left(\varphi^{n}\right)}\right) .
$$

We consider two cases: $v\left(\varphi^{n}\right) \rightarrow \infty$ and $v\left(\varphi^{n}\right) \nrightarrow \infty$. In the first case by Remark $14 w\left(\varphi^{n}\right) \rightarrow \infty$, as well. Then, for large $n, \operatorname{length}_{R}\left(R / \mathfrak{m}^{v\left(\varphi^{n}\right)}\right)$ and length $R\left(R / \mathfrak{m}^{w\left(\varphi^{n}\right)}\right)$ are polynomials of precise degree $d$ in $v\left(\varphi^{n}\right)$ and $w\left(\varphi^{n}\right)$, respectively, with highest degree terms $e(\mathfrak{m})\left(v\left(\varphi^{n}\right)\right)^{d} / d$ ! and $e(\mathfrak{m})\left(w\left(\varphi^{n}\right)\right)^{d} / d$ !. Thus, for large $n$

$$
\frac{e(\mathfrak{m})}{d !}\left(v\left(\varphi^{n}\right)\right)^{d} \leqslant \operatorname{length}\left(R / \varphi^{n}(\mathfrak{m}) R\right) \leqslant \frac{e(\mathfrak{m})}{d !}\left(w\left(\varphi^{n}\right)\right)^{d},
$$

which gives the result after applying logarithm, dividing by $n$ and taking limits. In the second case, when $v\left(\varphi^{n}\right) \nrightarrow \infty$, the sequence $\left\{v\left(\varphi^{n}\right)\right\}$ is bounded. Hence, there is a number $c$ such that $1 \leqslant v\left(\varphi^{n}\right) \leqslant c$. Applying logarithm, dividing by $n$ and taking limits, we get $v_{h}(\varphi)=0$. Now, if $w\left(\varphi^{n}\right) \rightarrow \infty$, then starting with the inequality

$$
1 \leqslant \operatorname{length}\left(R / \varphi^{n}(\mathfrak{m}) R\right) \leqslant \operatorname{length}_{R}\left(R / \mathfrak{m}^{w\left(\varphi^{n}\right)}\right)
$$

and repeating the same argument as before, we arrive at the desired inequality

$$
v_{h}(\varphi)=0 \leqslant h_{\mathrm{loc}}(\varphi) \leqslant d \cdot w_{h}(\varphi) .
$$

Finally if $w\left(\varphi^{n}\right) \nrightarrow \infty$, then the sequence $\left\{w\left(\varphi^{n}\right)\right\}$ is also bounded and there is a number $c^{\prime}$ such that $1 \leqslant w\left(\varphi^{n}\right) \leqslant c^{\prime}$. After applying logarithm, dividing by $n$ and taking limits, get $w_{h}(\varphi)=0$. Since $v_{h}(\varphi)=0$ as well, the proof will be completed by showing $h_{\mathrm{loc}}(\varphi, R)=0$. This follows from inequalities

$$
1 \leqslant \operatorname{length}\left(R / \varphi^{n}(\mathfrak{m}) R\right) \leqslant \operatorname{length}_{R}\left(R / \mathfrak{m}^{w\left(\varphi^{n}\right)}\right) \leqslant \operatorname{length}_{R}\left(R / \mathfrak{m}^{c^{\prime}}\right) .
$$

c) There is nothing to prove if $\delta=0$, so assume $\delta>0$. By Lemma 4 to say that $\varphi$ is contracting is equivalent to saying $\varphi^{\delta}(\mathfrak{m}) R \subseteq \mathfrak{m}^{2}$, which by definition gives $v\left(\varphi^{\delta}\right) \geqslant 2$. Hence, using parts a) and b) we obtain

$$
(\log 2) / \delta \leqslant\left(\log v\left(\varphi^{\delta}\right)\right) / \delta \leqslant \sup _{n}\left\{\left(\log v\left(\varphi^{n}\right)\right) / n\right\}=v_{h}(\varphi) \leqslant h_{\mathrm{loc}}(\varphi) / d .
$$


d) If $R$ is of characteristic $p$ and $\varphi$ is its Frobenius endomorphism, then by [29, Proposition 3.2]

$$
p^{n d} \leqslant \operatorname{length}\left(R / \varphi^{n}(\mathfrak{m}) R\right) \leqslant \min _{\left\{y_{1}, \ldots, y_{d}\right\}}\left[\operatorname{length}_{R}\left(R /\left(y_{1}, \ldots, y_{d}\right) R\right)\right] \cdot p^{n d},
$$

where $\left\{y_{1}, \ldots, y_{d}\right\}$ runs over all systems of parameters of $R$. Apply $\operatorname{logarithm}$, divide by $n$ and take limits to get $h_{\mathrm{loc}}(\varphi)=d \cdot \log p$.

Corollary 16. Let $(R, \mathfrak{m}, \varphi)$ be a local algebraic dynamical system. If $\operatorname{dim} R$ is zero, then $h_{\mathrm{loc}}(\varphi)=0$. Conversely, if $\varphi$ is contracting and $h_{\mathrm{loc}}(\varphi)=0$, then $\operatorname{dim} R$ is zero.

Proof: If $\operatorname{dim} R=0$, then $R$ is artinian and the result follows from inequalities $1 \leqslant \operatorname{length}\left(R / \varphi^{n}(\mathfrak{m} R)\right) \leqslant \operatorname{length}(R)<\infty$. The converse statement follows from part c) of Theorem 1 .

Local entropy can be computed using other methods. To show this we need a definition.

Definition 17. Let $R$ be a noetherian local ring, and let $\varphi$ be an endomorphism of $R$. Let R-Mod be the category of $R$-modules. For every $n \in \mathbb{N}$ we define a functor $\Phi^{n}: \mathrm{R}$-Mod $\rightarrow$ R-Mod as follows: if $M \in \mathrm{R}-\mathrm{Mod}$, then

$$
\Phi^{n}(M):=M \otimes_{R} \varphi_{*}^{n} R,
$$

where the $R$-module structure of $\Phi^{n}(M)$ is defined to be

$$
r \cdot x=\sum m_{i} \otimes r \cdot r_{i}, \quad \text { if } \quad x=\sum m_{i} \otimes r_{i} \in \Phi^{n}(M) \quad \text { and } \quad r \in R .
$$

For the Frobenius endomorphism the functors defined in Definition 17 are known as Frobenius functors. They were first introduced in [40, Definition 1.2]. Properties of Frobenius functors were established in [40] and [25]. The same proofs can be re-written for the functors $\Phi^{n}$. Thus, these functors share similar properties with Frobenius functors.

Proposition 18. Let $(R, \varphi)$ be a local algebraic dynamical system. If $M$ is a nonzero module of finite length, then

$$
h_{\mathrm{loc}}(\varphi)=\lim _{n \rightarrow \infty} \frac{1}{n} \cdot \log \operatorname{length}_{R}\left(\Phi^{n}(M)\right) .
$$

Proof: It is easy to show $\Phi^{n}(R / \mathfrak{m}) \cong R / \varphi^{n}(\mathfrak{m}) R$ (see [40, p. 54] or $[\mathbf{2 5}$, no. 2.6]). Thus,

$$
\operatorname{length}_{R}\left(\Phi^{n}(R / \mathfrak{m})\right)=\operatorname{length}_{R}\left(R / \varphi^{n}(\mathfrak{m}) R\right) .
$$


Since $M$ is of finite length, there is a surjection $M \rightarrow R / \mathfrak{m} \rightarrow 0$. Apply the functor $\Phi^{n}$ to obtain a surjection $\Phi^{n}(M) \rightarrow \Phi^{n}(R / \mathfrak{m}) \rightarrow 0$. Then using Proposition 12b)

$\operatorname{length}_{R}\left(\Phi^{n}(R / \mathfrak{m})\right) \leqslant \operatorname{length}_{R}\left(\Phi^{n}(M)\right) \leqslant \operatorname{length}\left(R / \varphi^{n}(\mathfrak{m}) R\right) \cdot \operatorname{length}_{R}(M)$.

The result follows after applying logarithm, dividing by $n$ and letting $n \rightarrow \infty$.

1.3. Properties of local entropy. Many of the properties that we will establish for local entropy in this section are also shared by topological entropy.

Proposition 19. Let $(R, \mathfrak{m}, \varphi)$ be a local algebraic dynamical system and let $r \in \mathbb{N}$. Then $h_{\mathrm{loc}}\left(\varphi^{r}\right)=r \cdot h_{\mathrm{loc}}(\varphi)$.

Proof: By definition of local entropy

$$
\begin{aligned}
h_{\mathrm{loc}}\left(\varphi^{r}\right) & =\lim _{n \rightarrow \infty}(1 / n) \cdot \log \operatorname{length}\left(R / \varphi^{r n}(\mathfrak{m}) R\right) \\
& =r \cdot \lim _{n \rightarrow \infty}(1 /(r n)) \cdot \log \operatorname{length}\left(R / \varphi^{r n}(\mathfrak{m}) R\right) \\
& =r \cdot h_{\mathrm{loc}}(\varphi) .
\end{aligned}
$$

Proposition 20. Let $f:(R, \mathfrak{m}, \varphi) \rightarrow(S, \mathfrak{n}, \psi)$ be a morphism between two local algebraic dynamical systems. Assume that $f$ is of finite length. Then

a) In general $h_{\mathrm{loc}}(\psi) \leqslant h_{\mathrm{loc}}(\varphi)$.

b) If in addition $f$ is flat, then $h_{\mathrm{loc}}(\psi)=h_{\mathrm{loc}}(\varphi)$.

Proof: a) Since $f$ is a morphism between local algebraic dynamical systems, $\psi^{n} \circ f=f \circ \varphi^{n}$. We use Lemma 15a)

$$
\begin{aligned}
\operatorname{length}\left(S / \psi^{n}(\mathfrak{n}) S\right) & \leqslant \operatorname{length}\left(\psi^{n} \circ f\right)=\operatorname{length}\left(f \circ \varphi^{n}\right) \\
& \leqslant \operatorname{length}(R / f(\mathfrak{m}) R) \cdot \operatorname{length}\left(R / \varphi^{n}(\mathfrak{m}) R\right) .
\end{aligned}
$$

Apply logarithm, divide by $n$ and take limits as $n$ approaches infinity.

b) If $f$ is flat, using Lemma 15a) and b) we obtain

$$
\begin{aligned}
\operatorname{length}\left(R / \varphi^{n}(\mathfrak{m}) R\right) & =\operatorname{length}\left(f \circ \varphi^{n}\right) / \operatorname{length}(R / f(\mathfrak{m}) R) \\
& =\operatorname{length}\left(\psi^{n} \circ f\right) / \operatorname{length}(R / f(\mathfrak{m}) R) \\
& \leqslant \operatorname{length}\left(S / \psi^{n}(\mathfrak{n}) S\right) .
\end{aligned}
$$

Apply logarithm, divide by $n$ and take limits as $n$ approaches infinity. For the inequality in the other direction we use part a). 
With regard to Proposition 20, C. Huneke has asked us the following question:

Question 21. Let $f:(R, \mathfrak{m}, \varphi) \rightarrow(S, \mathfrak{n}, \psi)$ be a morphism, not necessarily of finite length, between two local algebraic dynamical systems. The ideal $f(\mathfrak{m}) S$ is easily seen to be $\psi$-stable and $\psi$ induces an endomorphism $\bar{\psi}: S / f(\mathfrak{m}) S \rightarrow S / f(\mathfrak{m}) S$ that is of finite length. If $f$ is flat, does the equality $h_{\mathrm{loc}}(\psi)=h_{\mathrm{loc}}(\varphi)+h_{\mathrm{loc}}(\bar{\psi})$ hold?

One can see quickly that the inequality $h_{\mathrm{loc}}(\psi) \leqslant h_{\mathrm{loc}}(\varphi)+h_{\mathrm{loc}}(\bar{\psi})$ always holds even if $f$ is not flat.

Corollary 22. Let $(R, \mathfrak{m}, \varphi)$ be a local algebraic dynamical system. If $\widehat{R}$ is the $\mathfrak{m}$-adic completion of $R$ then $h_{\mathrm{loc}}(\varphi)=h_{\mathrm{loc}}(\hat{\varphi})$.

Proof: We have a flat morphism of finite length $\hat{\bullet}:(R, \varphi) \rightarrow(\hat{R}, \hat{\varphi})$. Apply Proposition 20.

Corollary 23. Consider homomorphisms of finite length $f:(R, \mathfrak{m}) \rightarrow$ $(S, \mathfrak{n})$ and $g:(S, \mathfrak{n}) \rightarrow(R, \mathfrak{m})$ of noetherian local rings. Then

$$
h_{\mathrm{loc}}(g \circ f)=h_{\mathrm{loc}}(f \circ g) \text {. }
$$

Proof: $f:(R, g \circ f) \rightarrow(S, f \circ g)$ and $g:(S, f \circ g) \rightarrow(R, g \circ f)$ are morphisms between local algebraic dynamical systems. By Proposition 20

$$
h_{\mathrm{loc}}(f \circ g) \leqslant h_{\mathrm{loc}}(g \circ f) \text { and } h_{\mathrm{loc}}(g \circ f) \leqslant h_{\mathrm{loc}}(f \circ g) \text {. }
$$

Corollary 24 (Invariance). Let $(R, \mathfrak{m})$ and $(S, \mathfrak{n})$ be noetherian local rings. Suppose $f: R \rightarrow S$ is an isomorphism, and let $\varphi$ be an endomorphism of of finite length of $R$. Then $h_{\mathrm{loc}}\left(f \circ \varphi \circ f^{-1}\right)=h_{\mathrm{loc}}(\varphi)$.

Proof: Apply Corollary 23 to homomorphisms $f \circ \varphi: R \rightarrow S$ and $f^{-1}: S \rightarrow$ $R$.

Corollary 25. Let $(R, \varphi)$ be a local algebraic dynamical system and let $\mathfrak{a}$ be a $\varphi$-stable ideal of $R$. Write $\bar{\varphi}$ and $\tilde{\varphi}$ for endomorphisms induced by $\varphi$ on $R / \mathfrak{a}$ and $R / \varphi(\mathfrak{a}) R$, respectively. Then $h_{\mathrm{loc}}(\bar{\varphi})=h_{\mathrm{loc}}(\tilde{\varphi})$.

Proof: Let $f: R / \mathfrak{a} \rightarrow R / \varphi(\mathfrak{a}) R$ and $g: R / \varphi(\mathfrak{a}) R \rightarrow R / \mathfrak{a}$ be homomorphisms induced by $\varphi$ and the identity map of $R$. Apply Corollary 23 .

The next two lemmas will be used in our proof of Proposition 28.

Lemma 26. Let $\left\{a_{n}\right\}$ and $\left\{b_{n}\right\}$ be two sequences of real numbers not less than 1 such that $\lim _{n \rightarrow \infty}\left(\log a_{n}\right) / n=\alpha$ and $\lim _{n \rightarrow \infty}\left(\log b_{n}\right) / n=\beta$ exist. Then

$$
\lim _{n \rightarrow \infty} \log \left(a_{n}+b_{n}\right) / n=\max \{\alpha, \beta\}
$$


Proof: See [1, p. 312].

Lemma 27. Let $(R, \mathfrak{m}, \varphi)$ be a local algebraic dynamical system. Let $\mathfrak{a}_{1}, \ldots, \mathfrak{a}_{s}$ be a collection of not necessarily distinct $\varphi$-stable ideals of $R$. Let $\bar{\varphi}$ and $\bar{\varphi}_{i}$ be the endomorphisms induced by $\varphi$ on $R / \prod_{i} \mathfrak{a}_{i}$ and $R / \mathfrak{a}_{i}$, respectively. Then

$$
h_{\mathrm{loc}}(\bar{\varphi})=\max \left\{h_{\mathrm{loc}}\left(\bar{\varphi}_{i}\right) \mid 1 \leqslant i \leqslant s\right\} .
$$

Proof: We proceed by induction on $s$, the number of ideals, counting possible repetitions. There is nothing to prove if $s=1$, so suppose $s=2$. We may assume $\mathfrak{a}_{1} \mathfrak{a}_{2}=0$; if not, we can replace $R$ with $R / \mathfrak{a}_{1} \mathfrak{a}_{2}$ without loss of generality. Then $\mathfrak{a}_{2}$ is a finitely generated $\left(R / \mathfrak{a}_{1}\right)$-module. Hence for some integer $r$, we have an exact sequence

$$
\left(R / \mathfrak{a}_{1}\right)^{r} \rightarrow R \rightarrow R / \mathfrak{a}_{2} \rightarrow 0 .
$$

Tensoring by $R / \varphi^{n}(\mathfrak{m}) R$ and taking lengths we get:

$$
\operatorname{length}\left(\varphi^{n}\right) \leqslant r \cdot \operatorname{length}\left(\bar{\varphi}_{1}^{n}\right)+\operatorname{length}\left(\bar{\varphi}_{2}^{n}\right) .
$$

Now let $n \rightarrow \infty$ and apply Lemma 26 to obtain

$$
h_{\mathrm{loc}}(\varphi) \leqslant \max \left\{h_{\mathrm{loc}}\left(\bar{\varphi}_{1}\right), h_{\mathrm{loc}}\left(\bar{\varphi}_{2}\right)\right\} .
$$

On the other hand by Proposition 20a) we know that $h_{\mathrm{loc}}(\varphi) \geqslant h_{\mathrm{loc}}\left(\bar{\varphi}_{i}\right)$, for each $i$. This establishes the case $s=2$. The general case follows easily by induction.

Our next result shows that if all minimal prime ideals of a noetherian local ring $R$ are stable under an endomorphism of the ring, then the local entropy is equal to the maximum local entropies of the endomorphisms induced on irreducible components of $\operatorname{Spec} R$.

Proposition 28. Let $(R, \mathfrak{m}, \varphi)$ be a local algebraic dynamical system. Suppose all minimal prime ideal of $R$ are $\varphi$-stable and for each $\mathfrak{p}_{i} \in$ Specmin $R$, let $\psi_{i}$ be the endomorphism induced by $\varphi$ on $R / \mathfrak{p}_{i}$. Then

$$
h_{\mathrm{loc}}(\varphi)=\max \left\{h_{\mathrm{loc}}\left(\psi_{i}\right) \mid \mathfrak{p}_{i} \in \operatorname{Specmin} R\right\} .
$$

Proof: Let $\operatorname{Specmin} R=\left\{\mathfrak{p}_{1}, \ldots, \mathfrak{p}_{s}\right\}$ and let $\mathfrak{a}=\prod_{i} \mathfrak{p}_{i}$. Then $\mathfrak{a}$ is contained in the nilradical of $R$, hence $\mathfrak{a}^{N}=(0)$ for some $N$, that is, $R=R / \mathfrak{a}^{N}$. Apply Lemma 27 to obtain

$$
h_{\mathrm{loc}}(\varphi)=\max \left\{h_{\mathrm{loc}}\left(\psi_{i}\right) \mid \mathfrak{p}_{i} \in \operatorname{Specmin} R\right\} .
$$

Remark 29. In Proposition 42 we will generalize equation (1). 
1.4. Reduction to equal characteristic. The main result in this section shows that computing local entropy in mixed characteristic can be reduced to the case of equal characteristic $p>0$.

For a given local algebraic dynamical system $(R, \mathfrak{m}, \varphi)$, we define

$$
S:=\bigcap_{n=1}^{\infty} \varphi^{n}(R) \text { and } \mathfrak{n}:=\bigcap_{n=1}^{\infty} \varphi^{n}(\mathfrak{m}) .
$$

Lemma 30. Let $(R, \mathfrak{m}, \varphi)$ be a local algebraic dynamical system. Let $S$ and $\mathfrak{n}$ be as defined in equation (2), and let $\mathfrak{a}$ be the ideal generated by $\mathfrak{n}$ in $R$. Then

a) $S$ is a local subring of $R$ with maximal ideal $\mathfrak{n}$.

b) a is a $\varphi$-stable ideal of $R$.

c) If $\varphi$ is in addition injective, then $\varphi(\mathfrak{a}) R=\mathfrak{a}$.

Proof: a) It is immediately clear that $S$ is a subring of $R$ and that $\mathfrak{n}$ is an ideal of $S$. To show that $\mathfrak{n}$ is the (only) maximal ideal of $S$, consider an element $s \in S \backslash \mathfrak{n}$. Since $s \notin \mathfrak{n}$, there is an $n_{0}$ such that $s \notin \varphi^{n_{0}}(\mathfrak{m})$. In fact, since for $n \geqslant n_{0}, \varphi^{n}(\mathfrak{m}) \subseteq \varphi^{n_{0}}(\mathfrak{m})$, we see that $s \notin \varphi^{n}(\mathfrak{m})$ for all $n \geqslant n_{0}$. Hence, there are units $y_{n} \in R \backslash \mathfrak{m}$ such that $s=\varphi^{n}\left(y_{n}\right)$ for all $n \geqslant n_{0}$. Since $s$ is clearly a unit in $R$, it has a unique multiplicative inverse $s^{-1}$ in $R$. From uniqueness of multiplicative inverse it immediately follows that we must have $s^{-1}=\varphi^{n}\left(y_{n}^{-1}\right)$, for all $n \geqslant n_{0}$. Hence, $s^{-1} \in S$, that is, $s$ is also a unit in $S$.

b) Note that by its definition, $\mathfrak{a}$ has a set of generators $x_{1}, \ldots, x_{g} \in \mathfrak{n}$. So $\varphi(\mathfrak{a}) R$ can be generated by $\varphi\left(x_{1}\right), \ldots, \varphi\left(x_{g}\right)$ and it suffices to show that each $\varphi\left(x_{i}\right)$ is in $\mathfrak{a}$. Since $x_{i} \in \mathfrak{n}$, there is a sequence of element $y_{i, n} \in$ $\mathfrak{m}$ such that $x_{i}=\varphi\left(y_{i, 1}\right)=\cdots=\varphi^{n}\left(y_{i, n}\right)=\cdots$. Thus, $\varphi\left(x_{i}\right)=$ $\varphi^{2}\left(y_{i, 1}\right)=\cdots=\varphi^{n+1}\left(y_{i, n}\right)=\cdots$, showing that $\varphi\left(x_{i}\right) \in \mathfrak{n} \subset \mathfrak{a}$.

c) Now suppose $\varphi$ is injective. To show $\varphi(\mathfrak{a}) R=\mathfrak{a}$ it suffices to show that each $x_{i}$ is in $\varphi(\mathfrak{a})$. Since $x_{i} \in \mathfrak{n}$, there is a sequence of element $y_{i, n} \in$ $\mathfrak{m}$ such that $x_{i}=\varphi\left(y_{i, 1}\right)=\cdots=\varphi^{n}\left(y_{i, n}\right)=\cdots$. Since $x_{i}=\varphi\left(y_{i, 1}\right)$, we will be done by showing that $y_{i, 1} \in \mathfrak{n}$. By injectivity of $\varphi, y_{i, 1}=$ $\varphi\left(y_{i, 2}\right)=\cdots=\varphi^{n-1}\left(y_{i, n}\right)=\cdots$, which means $y_{i, 1} \in \mathfrak{n}$.

Remark 31. Let $(R, \mathfrak{m}, \varphi)$ be a local algebraic dynamical system and let $\mathfrak{n}$ be as defined in equation (2). If $\mathfrak{n}=(0)$, then by Lemma 30 the ring $R$ contains a field and is of equal characteristic. As noted in [2, Remark 5.9, p. 10], this occurs, for example, if $\varphi$ is a contracting endomorphism.

Proposition 32. Let $(R, \mathfrak{m}, \varphi)$ be a local algebraic dynamical system. Let $\mathfrak{a}$ be the ideal of $R$ defined in Lemma 30, and let $\bar{\varphi}$ be the endomorphism induced by $\varphi$ on $R / \mathfrak{a}$. Then 
a) $h_{\mathrm{loc}}(\bar{\varphi})=h_{\mathrm{loc}}(\varphi)$.

b) If $R$ is of mixed characteristic, then $R / \mathfrak{a}$ is of equal characteristic $p>0$.

Proof: a) Note that $\varphi^{n}(\mathfrak{m}) R \supset \mathfrak{a}$ for all $n \geqslant 1$. Hence $\varphi^{n}(\mathfrak{m}) R+\mathfrak{a}=$ $\varphi^{n}(\mathfrak{m}) R$, showing that length $\left(R /\left(\varphi^{n}(\mathfrak{m}) R+\mathfrak{a}\right)\right)=\operatorname{length}\left(R / \varphi^{n}(\mathfrak{m}) R\right)$, giving the result.

b) With reference to Lemma 30, the image of the subring $S$ of $R$ in $R / \mathfrak{a}$ is a field, because it's maximal ideal $\mathfrak{n}$ is contained in $\mathfrak{a}$ and is mapped to 0 . Hence $R / \mathfrak{a}$ contains a field and must be a local ring of equal characteristic $p>0$, as its residue field is of characteristic $p>0$.

1.5. Local entropy and degree. The analogy between local and topological entropies also extends to their relation to degree. Misiurewicz and Przytycki showed in [36], that if $f$ is a $C^{1}$ self-map of a smooth compact orientable manifold $M$, then

$$
h_{\text {top }}(f) \geqslant \log |\operatorname{deg}(f)| .
$$

For a holomorphic self-morphism $f$ of $\mathbb{P}^{n}(\mathbb{C})$, Gromov showed in $[\mathbf{2 3}]$

$$
h_{\text {top }}(f)=\log |\operatorname{deg}(f)| \text {. }
$$

Here $\operatorname{deg}(f)$ is the topological degree of $f$.

In this section we obtain similar formulas for finite endomorphisms of local domains, relating their local entropy to degree. For local CohenMacaulay domains we prove an analog of Gromov's formula.

Definition 33. Let $f: R \rightarrow S$ be a finite homomorphism of noetherian local rings. Assume that $R$ is a domain. Then by degree of $f, \operatorname{deg}(f)$, we mean the rank of the $R$-module $f_{*} S$.

Lemma 34. Let $f:(R, \mathfrak{m}) \rightarrow(S, \mathfrak{n})$ be a finite homomorphism of noetherian local rings with residue fields $k_{R}$ and $k_{S}$, respectively. Assume that $R$ is a domain. Let $\mathfrak{q}$ be an $\mathfrak{m}$-primary ideal of $R$. Then

$$
e_{S}(f(\mathfrak{q}) S)=\frac{e_{R}(\mathfrak{q}) \cdot \operatorname{deg}(f)}{\left[f_{*} k_{S}: k_{R}\right]}
$$


Proof: Let $d=\operatorname{dim} R=\operatorname{dim} S$. By definition of multiplicity and properties of length we quickly obtain

$$
\begin{aligned}
e_{R}\left(\mathfrak{q}, f_{*} S\right) & =\lim _{m \rightarrow \infty} \frac{d !}{m^{d}} \cdot \operatorname{length}_{R}\left(f_{*}\left(\frac{S}{(f(\mathfrak{q}) S)^{m}}\right)\right) \\
& =\left[f_{*} k_{S}: k_{R}\right] \cdot \lim _{m \rightarrow \infty} \frac{d !}{m^{d}} \cdot \operatorname{length}_{S}\left(\frac{S}{(f(\mathfrak{q}) S)^{m}}\right) \\
& =\left[f_{*} k_{S}: k_{R}\right]^{n} \cdot e_{S}(f(\mathfrak{q}) S) .
\end{aligned}
$$

On the other hand $e_{R}\left(\mathfrak{q}, f_{*} S\right)=e_{R}(\mathfrak{q}) \cdot \operatorname{deg}(f)$ (see [35, Theorem 14.8]), and equation (3) follows.

Corollary 35. Let $(R, \mathfrak{m}, \varphi)$ be a local algebraic dynamical system, where $R$ is a domain and $\varphi$ is finite, and let $k$ be the residue field of $R$. Set $d:=$ $\operatorname{dim} R$ and define $q_{\varphi}:=\exp \left(h_{\mathrm{loc}}(\varphi, R) / d\right)$. For elements $x_{1}, \ldots, x_{d} \in \mathfrak{m}$ let $\chi\left(x_{1}, \ldots, x_{d} ; R\right)$ be the Euler-Poincaré characteristic of the Koszul complex on these elements. The following conditions are equivalent:

a) $\log \operatorname{deg}(\varphi)=\log \left[\varphi_{*} k: k\right]+h_{\operatorname{loc}}(\varphi)$.

b) For any system of parameters $\left\{x_{1}, \ldots, x_{d}\right\}$ of $R$ and for any $n \in \mathbb{N}$

$$
\chi\left(\varphi^{n}\left(x_{1}\right), \ldots, \varphi^{n}\left(x_{d}\right) ; R\right)=q_{\varphi}^{n d} \cdot \chi\left(x_{1}, \ldots, x_{d} ; R\right) .
$$

c) Equation (4) holds for some system of parameters of $R$ and some $n \in \mathbb{N}$.

Proof: Let $\left\{x_{1}, \ldots, x_{d}\right\}$ be a system of parameters of $R$ and let $\mathfrak{q}$ be the $\mathfrak{m}$-primary ideal that they generate. By $[\mathbf{4 5}$, Chapter IV, Theorem 1]

$$
e(\mathfrak{q})=\chi\left(x_{1}, \ldots, x_{d} ; R\right) .
$$

Since $\left\{\varphi^{n}\left(x_{1}\right), \ldots, \varphi^{n}\left(x_{d}\right)\right\}$ is also a system of parameters of $R$, the result quickly follows from equation (3) in Lemma 34 .

Example 36. Let $(R, \mathfrak{m})$ be a noetherian local domain of prime characteristic $p$, and let $\varphi$ be the Frobenius endomorphism of $R$. Then by [30, Proposition 2.3] condition a) of Corollary 35 holds.

Remark 37. When $f:\left(R, \mathfrak{m}, k_{R}\right) \rightarrow\left(S, \mathfrak{n}, k_{S}\right)$ is a finite homomorphism of local rings, then $\left[f_{*} k_{S}: k_{R}\right] \cdot \operatorname{length}_{S}(S / \mathfrak{m} S)$ is the minimal number of generators of $f_{*} S$ over $R$, by Nakayama lemma.

Proposition 38. Let $(R, \mathfrak{m}, \varphi)$ be a local algebraic dynamical system, where $\varphi$ is finite, $R$ is a domain and $\operatorname{dim} R=d$. Let $k$ be the residue field of $R$. Then

a) $d \cdot v_{h}(\varphi) \leqslant \log \left(\operatorname{deg}(\varphi) /\left[\varphi_{*} k: k\right]\right) \leqslant h_{\operatorname{loc}}(\varphi)$.

b) If in addition $R$ is Cohen-Macaulay, $\log \left(\operatorname{deg}(\varphi) /\left[\varphi_{*} k: k\right]\right)=h_{\mathrm{loc}}(\varphi)$. 
Proof: a) By Theorem 1 we know $v_{h}(\varphi)=\sup _{n}\left\{\left(\log v\left(\varphi^{n}\right)\right) / n\right\}$. Hence, to show the first inequality it suffices to show

$$
v\left(\varphi^{n}\right)^{d / n} \leqslant \operatorname{deg}(\varphi) /\left[\varphi_{*} k: k\right] .
$$

Since $\varphi^{n}(\mathfrak{m}) R \subset \mathfrak{m}^{v\left(\varphi^{n}\right)}$, with the aid of Lemma 34 we obtain

$$
e(\mathfrak{m}) v\left(\varphi^{n}\right)^{d}=e\left(\mathfrak{m}^{v\left(\varphi^{n}\right)}\right) \leqslant e\left(\varphi^{n}(\mathfrak{m}) R\right)=\frac{e(\mathfrak{m}) \cdot \operatorname{deg}\left(\varphi^{n}\right)}{\left[\varphi_{*}^{n} k: k\right]} .
$$

For the second inequality, let $\mu\left(\varphi_{*}^{n} R\right)$, or simply $\mu$, be the minimum number of generators of the $R$-module $\varphi_{*}^{n} R$. Localizing the surjection $R^{\mu} \rightarrow \varphi_{*}^{n} R$ at $(0)$ we see $\operatorname{rank} \varphi_{*}^{n} R \leqslant \mu\left(\varphi_{*}^{n} R\right)$. On the other hand, as mentioned in Remark 37, we have $\mu\left(\varphi_{*}^{n} R\right)=\left[\varphi_{*} k: k\right]^{n}$. length $\left(R / \varphi^{n}(\mathfrak{m}) R\right)$. Since by definition of degree, $\operatorname{rank} \varphi_{*}^{n} R=\operatorname{deg}\left(\varphi^{n}\right)=$ $(\operatorname{deg}(\varphi))^{n}$, we conclude

$$
(\operatorname{deg}(\varphi))^{n} /\left[\varphi_{*} k: k\right]^{n} \leqslant \operatorname{length}\left(R / \varphi^{n}(\mathfrak{m}) R\right) .
$$

Apply logarithm, divide by $n$ and take limits as $n \rightarrow \infty$.

b) Let $\mathfrak{q}$ be an arbitrary parameter ideal of $R$. Then

$$
\operatorname{length}\left(R / \varphi^{n}(\mathfrak{m}) R\right) \leqslant \operatorname{length}\left(R / \varphi^{n}(\mathfrak{q}) R\right) .
$$

If $R$ is Cohen-Macaulay, then length $\left(R / \varphi^{n}(\mathfrak{q}) R\right)=e\left(\varphi^{n}(\mathfrak{q}) R\right)$ (see, e.g., [35, Theorem 17.11]). Thus, using Lemma 34

$$
\operatorname{length}\left(R / \varphi^{n}(\mathfrak{m}) R\right) \leqslant e\left(\varphi^{n}(\mathfrak{q}) R\right)=\frac{e(\mathfrak{q})(\operatorname{deg}(\varphi))^{n}}{\left[\varphi_{*} k: k\right]^{n}} .
$$

Applying logarithm, dividing by $n$, and taking limits as $n \rightarrow \infty$ we obtain

$$
h_{\mathrm{loc}}(\varphi) \leqslant \log \left(\operatorname{deg}(\varphi) /\left[\varphi_{*} k: k\right]\right) .
$$

This inequality together with the inequality in a) give the desired equality.

\subsection{Entropy at the vertex of the cone over a projective variety.} The following result, which will be used in this section was proved in $[\mathbf{5}$, Theorem 2.1] by Bhatnagar and the third author over infinite fields and in [41, Theorem 1.4] by Poonen over finite fields.

Theorem. Let $X$ be a projective variety defined over a field $k, \imath: X \hookrightarrow$ $\mathbb{P}_{k}^{N}$ a given embedding and $\mathcal{L}:=\imath^{*} \mathcal{O}_{\mathbb{P}_{k}^{N}}(1)$. Let $\varphi: X \rightarrow X$ be a selfmorphism that is polarized by $\mathcal{L}$, that is, $\varphi^{*}(\mathcal{L}) \cong \mathcal{L}^{\otimes q}$ for an integer $q \geqslant 2$. Then there exists a positive integer $r$ and a finite morphism $\mathbb{P}_{k}^{N} \rightarrow \mathbb{P}_{k}^{N}$ extending $\varphi^{r}$, where $N+1=\operatorname{dim}_{k} \mathrm{H}^{0}(X, \mathcal{L})$. 
Keeping the notation as above, let $\mathfrak{a}$ be the largest homogeneous ideal in $k\left[X_{0}, \ldots, X_{N}\right]$ defining $\imath(X)$ and let $R:=k\left[X_{0}, \ldots, X_{N}\right] / \mathfrak{a}$. Also let $R^{(q)}:=\bigoplus_{n \geqslant 0} R_{n q}$ be the $q$ th Veronese subring of $R$. Using the above theorem, we can fix an $r \in \mathbb{N}$ such that $\varphi^{r}$ extends to a finite selfmorphism $\mathbb{P}_{k}^{N} \rightarrow \mathbb{P}_{k}^{N}$. The proof of this theorem in [5], [41] shows that this self-morphism is given by $N+1$ forms $F_{0}, \ldots, F_{N}$ of degree $q^{r}$ that have no non-trivial common zeros in the algebraic closure of $k$. The assignment $X_{i} \mapsto F_{i}$ defines a finite endomorphism $\psi: R \rightarrow R$ that can be factored as

$$
R \rightarrow R^{\left(q^{r}\right)} \hookrightarrow R,
$$

where $R^{\left(q^{r}\right)} \hookrightarrow R$ is inclusion and $R \rightarrow R^{\left(q^{r}\right)}$ is a graded homomorphism that induces $\varphi^{r}$ on $X=\operatorname{Proj} R$. We want to calculate the local entropy of the local endomorphism induced by $\psi$ at the vertex of the affine cone Spec $R$ over $X$.

Remark 39. While the self-morphism $\mathbb{P}_{k}^{N} \rightarrow \mathbb{P}_{k}^{N}$ obtained from the theorem is not unique, the endomorphism $\psi: R \rightarrow R$ is unique up to a scalar multiple.

Proposition 40. Let $X$ be an integral projective variety of dimension d over a field $k$ with a given embedding $\imath: X \hookrightarrow \mathbb{P}_{k}^{N}$ and set $\mathcal{L}:=\imath^{*} \mathcal{O}_{\mathbb{P}_{k}^{N}}(1)$. Let $\varphi: X \rightarrow X$ be a self-morphism and assume that $\varphi^{*}(\mathcal{L}) \cong \mathcal{L}^{\otimes q}$ for an integer $q \geqslant 2$. Let $r, R$ and $\psi$ be as defined in the previous paragraph and let $h_{\mathrm{loc}}(\psi)$ be the local entropy of $\psi$ at the vertex of the affine cone Spec $R$ over $X$. Then

$$
h_{\mathrm{loc}}(\psi)=\log \operatorname{deg}(\psi)=(d+1) \log q^{r} .
$$

Proof: By Theorem 1b) and Proposition 38a) it suffices to show $v_{h}(\psi) \geqslant$ $\log q^{r}$ and $w_{h}(\psi) \leqslant \log q^{r}$. For the first inequality, note that as discussed prior to this proposition, $\psi$ is induced by assignments $X_{i} \mapsto F_{i}$ for $N+1$ homogeneous forms $F_{0}, \ldots, F_{N}$ of degree $q^{r}$ in the variables $X_{i}$. Thus, $\psi^{n}$ is given by forms of degree $q^{n r}$. This shows, with notations of Theorem 1 , that $v\left(\psi^{n}\right) \geqslant q^{n r}$. Hence

$$
v_{h}(\psi)=\lim _{n \rightarrow \infty} \frac{1}{n} \log v\left(\psi^{n}\right) \geqslant \log q^{r} .
$$

To prove the second inequality we use elimination theory: by $[\mathbf{3 1}$, Corollary to Theorem 1, p. 169] we get $\left\langle X_{0}, \ldots, X_{N}\right\rangle^{s} \subset\left\langle F_{0}, \ldots, F_{N}\right\rangle$, where $s=q^{r}(N+1)-N$. Thus, $w\left(\psi^{n}\right) \leqslant q^{n r}(N+1)-N$ and we obtain

$$
w_{h}(\psi)=\lim _{n \rightarrow \infty} \frac{1}{n} \log w\left(\psi^{n}\right) \leqslant \log q^{r} .
$$

This concludes the proof. 
1.7. Integral endomorphisms. In this section we study local algebraic dynamical systems $(R, \varphi)$ generated by integral endomorphisms. We begin with a simple observation. Let $(R, \varphi)$ be a local algebraic dynamical system. Then for every $n \in \mathbb{N}$,

$$
\varphi\left(\operatorname{ker} \varphi^{n}\right) \subset \operatorname{ker} \varphi^{n-1} \subset \operatorname{ker} \varphi^{n} .
$$

Hence $\varphi$ induces a local endomorphism of $R / \operatorname{ker} \varphi^{n}$.

Proposition 41. Let $(R, \mathfrak{m}, \varphi)$ be a local algebraic dynamical system. Let $\bar{\varphi}_{n}$ be the local endomorphism induced by $\varphi$ on $R / \operatorname{ker} \varphi^{n}, n \in \mathbb{N}$. Then

a) $h_{\mathrm{loc}}(\varphi)=h_{\mathrm{loc}}\left(\bar{\varphi}_{n}\right)$.

b) If $\varphi$ is integral, then so is $\bar{\varphi}_{n}$.

c) If $n$ is large enough, then $\bar{\varphi}_{n}: R / \operatorname{ker} \varphi^{n} \rightarrow R / \operatorname{ker} \varphi^{n}$ is injective.

Proof: a) Apply Corollary 25 to the endomorphism $\varphi^{n}$ of $R$, taking $\operatorname{ker} \varphi^{n}$ as the ideal $\mathfrak{a}$ in that corollary. Since $\varphi^{n}\left(\operatorname{ker} \varphi^{n}\right) R=(0)$, by that corollary $h_{\text {loc }}\left(\bar{\varphi}_{n}^{n}\right)=h_{\text {loc }}\left(\varphi^{n}\right)$. Now use Proposition 19 .

b) This is clear (see [8, Chapter V, Proposition 2, p. 305]).

c) $R$ is noetherian, so the ascending chain $\operatorname{ker} \varphi \subset \operatorname{ker} \varphi^{2} \subset \operatorname{ker} \varphi^{3} \subset \ldots$ is stationary. Let $n_{0}$ be such that $\operatorname{ker} \varphi^{n}=\operatorname{ker} \varphi^{n+1}$ for $n \geqslant n_{0}$. We will show that if $n \geqslant n_{0}$, then $\bar{\varphi}_{n}: R / \operatorname{ker} \varphi^{n} \rightarrow R / \operatorname{ker} \varphi^{n}$ is injective. Let $\bar{x} \in R / \operatorname{ker} \varphi^{n}$. Saying $\bar{\varphi}_{n}(\bar{x})=0$ is equivalent to saying $\varphi(x) \in \operatorname{ker} \varphi^{n}$, which is equivalent to saying $x \in \operatorname{ker} \varphi^{n+1}$. Since $\operatorname{ker} \varphi^{n+1}=\operatorname{ker} \varphi^{n}$, we see that $x \in \operatorname{ker} \varphi^{n}$, or $\bar{x}=0$ in $R / \operatorname{ker} \varphi^{n}$. Thus, $\bar{\varphi}_{n}$ is injective.

Proposition 42. Let $(R, \mathfrak{m}, \varphi)$ be a local algebraic dynamical system, where $\varphi$ is integral. Let $\mathfrak{a}$ be the ideal obtained as the stable limit of the ascending chain of ideals $\operatorname{ker} \varphi \subset \operatorname{ker} \varphi^{2} \subset \operatorname{ker} \varphi^{3} \subset \cdots$. Write ${ }^{a} \varphi$ for the self-morphism of $\operatorname{Spec} R$ induced by $\varphi$. Then

a) ${ }^{a} \varphi$ permutes the minimal prime ideals of $\mathfrak{a}$.

b) Every minimal prime ideal of $\mathfrak{a}$ is in $\operatorname{Specmin} R$.

c) Let $\bar{\varphi}: R / \mathfrak{a} \rightarrow R / \mathfrak{a}$ be the endomorphism induced by $\varphi$. Assume that the permutation in part a) is of order $p$. For a minimal prime ideal $\mathfrak{p}_{i}$ of $\mathfrak{a}$ let $\psi_{i}$ be the endomorphism induced by $\varphi^{p}$ on $R / \mathfrak{p}_{i}$. Then

$$
h_{\mathrm{loc}}(\varphi)=\frac{1}{p} \cdot \max \left\{h_{\mathrm{loc}}\left(\psi_{i}\right) \mid \mathfrak{p}_{i} \text { is a minimal prime of } \mathfrak{a}\right\} \text {. }
$$


Proof: We recall that if a ring $S$ is integral over a subring $R$, then over every prime ideal $\mathfrak{p}$ of $R$ there lies a prime ideal $\mathfrak{q}$ of $S$. Moreover, if $\mathfrak{p}$ is a minimal prime ideal, then so is $\mathfrak{q}$ (see [35, Theorem 9.3]).

a) By Proposition 41, the endomorphism $\bar{\varphi}: R / \mathfrak{a} \rightarrow R / \mathfrak{a}$ is integral and injective. Apply the fact that we recalled above and note that $\mathfrak{a}$ has only a finite number of minimal prime ideals. (Any surjective map from a finite set to itself is also injective.)

b) Let $n$ be large enough so that $\operatorname{ker} \varphi^{n}=\mathfrak{a}$. Then $\varphi^{n}$ induces an integral injection $\tilde{\varphi}_{n}: R / \mathfrak{a} \hookrightarrow R$ and we have a commuting diagram

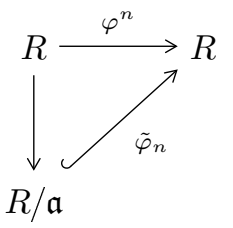

If $\mathfrak{q}$ is a minimal prime ideal of $\mathfrak{a}$, then by part a) (applied to $\varphi^{n}$ ) the ideal $\mathfrak{p}:=\left(\varphi^{n}\right)^{-1}(\mathfrak{q})$ is a minimal prime of $\mathfrak{a}$. Thus, $\left(\tilde{\varphi}_{n}\right)^{-1}(\mathfrak{q})=\mathfrak{p} / \mathfrak{a}$. We apply the fact that we recalled at the beginning of the proof to the ring injection $\tilde{\varphi}_{n}: R / \mathfrak{a} \hookrightarrow R$ and conclude $\mathfrak{q} \in \operatorname{Specmin} R$.

c) Let $n$ be large enough so that $\operatorname{ker} \varphi^{n p}=\mathfrak{a}$. By Proposition 41a) we know that $h_{\mathrm{loc}}\left(\varphi^{n p}\right)=h_{\mathrm{loc}}\left(\bar{\varphi}^{n p}\right)$. Since all minimal prime ideals of $R / \mathfrak{a}$ are $\bar{\varphi}^{n p}$-stable, by Proposition 28 we obtain

$$
h_{\mathrm{loc}}\left(\bar{\varphi}^{n p}\right)=\max \left\{h_{\mathrm{loc}}\left(\psi_{i}^{n}\right) \mid \mathfrak{p}_{i} \text { is a minimal prime of } \mathfrak{a}\right\} .
$$

Now an application of Proposition 19 quickly concludes the proof.

Corollary 43. Let $(R, \varphi)$ be a local algebraic dynamical system. Assume that $\varphi$ is integral and $\operatorname{Spec} R=V(\operatorname{ker} \varphi)$. Then

a) ${ }^{a} \varphi$ permutes the minimal prime ideals of $R$.

b) If $\mathfrak{p} \notin \operatorname{Specmin} R$ is a prime ideal of $R$, then $\varphi^{-1}(\mathfrak{p}) \notin \operatorname{Specmin} R$.

c) An element $x \in R$ belongs to a minimal prime ideal of $R$, if and only if $\varphi(x)$ belongs to a minimal prime ideal of $R$.

d) Assume that the permutation in part a) is of order $p$. For $\mathfrak{p}_{i} \in$ $\operatorname{Specmin} R$ let $\psi_{i}$ be the endomorphism induced by $\varphi^{p}$ on $R / \mathfrak{p}_{i}$. Then

$$
h_{\mathrm{loc}}(\varphi)=\frac{1}{p} \cdot \max \left\{h_{\mathrm{loc}}\left(\psi_{i}\right) \mid \mathfrak{p}_{i} \in \operatorname{Specmin} R\right\} .
$$

Proof: a) Since $\varphi$ is integral, image $\left({ }^{a} \varphi\right)=V(\operatorname{ker} \varphi)$. Thus, from the hypothesis $V(\operatorname{ker} \varphi)=\operatorname{Spec} R$ we see that ${ }^{a} \varphi$ is surjective. Hence, ${ }^{a} \varphi^{n}$ is 
also surjective for every $n \in \mathbb{N}$, i.e., $V\left(\operatorname{ker} \varphi^{n}\right)=\operatorname{image}\left({ }^{a} \varphi^{n}\right)=\operatorname{Spec} R$. So for every $n \in \mathbb{N}$, the set of minimal prime ideals of $\operatorname{ker} \varphi^{n}$ is equal to Specmin $R$. Take $n$ large enough and apply Proposition 42a).

b) Suppose $\mathfrak{q}:=\varphi^{-1}(\mathfrak{p}) \in \operatorname{Specmin} R$. If $\mathfrak{p}$ is not minimal, it contains a minimal prime ideal $\mathfrak{p}^{\prime}$. Moreover, $\operatorname{ker} \varphi \subset \mathfrak{p}^{\prime}$. By part a), $\varphi^{-1}\left(\mathfrak{p}^{\prime}\right) \in \operatorname{Specmin} R$. Since $\mathfrak{q} \supseteq \varphi^{-1}\left(\mathfrak{p}^{\prime}\right)$ and $\mathfrak{q}$ is minimal, we must have $\mathfrak{q}=\varphi^{-1}\left(\mathfrak{p}^{\prime}\right)$. This is a contradiction, because there can be no inclusion between prime ideals that lie over $\mathfrak{q}$ in the integral ring inclusion $\varphi:(R / \operatorname{ker} \varphi) \hookrightarrow R$.

c) Let $x$ be an element of $R$. If $\varphi(x) \in \mathfrak{p}$ for some $\mathfrak{p} \in \operatorname{Specmin} R$, then $x \in \varphi^{-1}(\mathfrak{p})$. By part a), $\varphi^{-1}(\mathfrak{p}) \in \operatorname{Specmin} R$. Conversely, suppose $x \in \mathfrak{q}$ for some $\mathfrak{q} \in \operatorname{Specmin} R$. Then by part a) there is a $\mathfrak{p} \in \operatorname{Specmin} R$ such that $\mathfrak{q}=\varphi^{-1}(\mathfrak{p})$. Hence $\varphi(x) \in \mathfrak{p}$.

d) Since all minimal prime ideals of $R$ are $\varphi^{p}$-stable, by Proposition 28, $h_{\text {loc }}\left(\varphi^{p}\right)=\max \left\{h_{\mathrm{loc}}\left(\psi_{i}\right) \mid \mathfrak{p}_{i} \in \operatorname{Specmin} R\right\}$. The result quickly follows from Proposition 19.

\section{Regularity, flatness and entropy}

In the second part of this paper we will present proofs of Theorems 2 and 3 . Let $(R, \mathfrak{m})$ be a noetherian local ring of positive prime characteristic $p$ and dimension $d$, and let $\varphi$ be the Frobenius endomorphism of $R$. In $[\mathbf{2 9}]$ Kunz showed that the following conditions are equivalent:

a) $R$ is regular.

b) $\varphi: R \rightarrow R$ is flat.

c) length $(R / \varphi(\mathfrak{m}) R)=p^{d}$.

d) length $\left(R / \varphi^{n}(\mathfrak{m}) R\right)=p^{n d}$ for some $n \in \mathbb{N}$.

Later Rodicio showed in $[\mathbf{4 2}]$, that these conditions are also equivalent to

e) flat $\operatorname{dim}_{R} \varphi_{*} R<\infty$.

At first glance, Kunz' conditions c) and d) may appear to be stated in terms of the characteristic $p$ of the ring and one may not expect to be able to extend, or even state them in arbitrary characteristic. Nevertheless, local entropy can be used to make sense of Kunz' numerical conditions c) and d) for all endomorphisms of finite length in any characteristic. Theorem 2 states that with this new interpretation, all conditions in Kunz' result are equivalent.

We should note that in [3, Theorem 13.3] Avramov, Iyengar and Miller have extended the equivalence of conditions a) and b) of Kunz and e) 
of Rodicio to arbitrary contracting local endomorphisms of noetherian local rings.

We list two results here that we will need in our proof of Theorem 2 .

Lemma 44 ([25, Lemma 3.2]). Let $(R, \mathfrak{m})$ be a noetherian local ring, and let $M$ be a finitely generated $R$-module. Consider an ideal $\mathfrak{b} \subseteq \mathfrak{m}$ of $R$. Then there exists an integer $\mu_{0} \geqslant 0$ such that $\operatorname{depth}\left(\mathfrak{m}, \mathfrak{b}^{\mu} M\right)>0$ for all $\mu \geqslant \mu_{0}$.

Remark 45. In using Lemma 44 we must pay particular attention to the standard convention that the depth of the zero module is $\infty$ (see, e.g., [26, p. 291]). Otherwise, if $M$ is an $R$-module of finite length, then for $\mu \gg 0$ we have $\mathfrak{m}^{\mu} M=(0)$, and this would have been a counterexample to Lemma 44.

The next proposition is taken from [9, Chapter 10, $\S 1$, Proposition 1].

Proposition 46. Let $R$ be a noetherian ring and let $\mathfrak{a}$ be an ideal of $R$. Let $0 \rightarrow M^{\prime} \rightarrow M \rightarrow M^{\prime \prime} \rightarrow 0$ be an exact sequence of $R$-modules. If we define $d^{\prime}=\operatorname{depth}\left(\mathfrak{a}, M^{\prime}\right), d=\operatorname{depth}(\mathfrak{a}, M)$, and $d^{\prime \prime}=\operatorname{depth}\left(\mathfrak{a}, M^{\prime \prime}\right)$, then one of the following mutually exclusive possibilities holds:

$$
d^{\prime}=d \leqslant d^{\prime \prime} \text { or } d=d^{\prime \prime}<d^{\prime} \text { or } d^{\prime \prime}=d^{\prime}-1<d \text {. }
$$

2.1. Kunz' regularity criterion via local entropy. Before we give the proof of Theorem 2 we need to establish two lemmas. We begin with a flatness criterion that is due to Nagata. A proof can be found in $[\mathbf{3 8}$, Chapter II, Theorem 19.1]. See also [35, Exercise 22.1, p. 178].

Theorem (Nagata). Let $g:(R, \mathfrak{m}) \rightarrow(S, \mathfrak{n})$ be an injective homomorphism of finite length of noetherian local rings. Then $S$ is flat over $R$, if and only if for every $\mathfrak{m}$-primary ideal $\mathfrak{q}$ of $R$,

$$
\operatorname{length}_{R}(R / \mathfrak{q}) \cdot \operatorname{length}_{S}(S / g(\mathfrak{m}) S)=\operatorname{length}_{S}(S / g(\mathfrak{q}) S) .
$$

We need a stronger version of Nagata's criterion that we state and prove here.

Lemma 47. Let $g:(R, \mathfrak{m}) \rightarrow(S, \mathfrak{n})$ be a homomorphism of finite length of noetherian local rings. If equation (6) holds for a family of $\mathfrak{m}$-primary ideals $\left\{\mathfrak{q}_{\alpha}\right\}_{\alpha \in A}$ that define the $\mathfrak{m}$-adic topology, then it holds for all $\mathfrak{m}$-primary ideals.

Proof: Let $\mathfrak{q}$ be an $\mathfrak{m}$-primary ideal. We will show equation (6) holds for $\mathfrak{q}$. First, using Proposition 12

$\operatorname{length}_{S}(S / g(\mathfrak{q}) S)=\operatorname{length}_{S}\left(S \otimes_{R} R / \mathfrak{q}\right) \leqslant \operatorname{length}_{S}(S / g(\mathfrak{m}) S) \cdot \operatorname{length}_{R}(R / \mathfrak{q})$. 
To show the reverse inequality, note that by assumption there is a $\mathfrak{q}_{\alpha} \subseteq \mathfrak{q}$. The exact sequence $0 \rightarrow \mathfrak{q} / \mathfrak{q}_{\alpha} \rightarrow R / \mathfrak{q}_{\alpha} \rightarrow R / \mathfrak{q} \rightarrow 0$ yields

$$
\operatorname{length}_{R}\left(R / \mathfrak{q}_{\alpha}\right)=\operatorname{length}_{R}(R / \mathfrak{q})+\operatorname{length}_{R}\left(\mathfrak{q} / \mathfrak{q}_{\alpha}\right) .
$$

If we tensor the previous exact sequence with $S$, we obtain an exact sequence of $S$-modules $\left(\mathfrak{q} / \mathfrak{q}_{\alpha}\right) \otimes_{R} S \rightarrow S / g\left(\mathfrak{q}_{\alpha}\right) S \rightarrow S / g(\mathfrak{q}) S \rightarrow 0$. Thus

$$
\operatorname{length}_{S}\left(S / g\left(\mathfrak{q}_{\alpha}\right) S\right) \leqslant \operatorname{length}_{S}(S / g(\mathfrak{q}) S)+\operatorname{length}_{S}\left(\left(\mathfrak{q} / \mathfrak{q}_{\alpha}\right) \otimes_{R} S\right) .
$$

Since equation (6) holds for $\mathfrak{q}_{\alpha}$, and using Proposition 12 we quickly see $\operatorname{length}_{R}\left(R / \mathfrak{q}_{\alpha}\right) \cdot \operatorname{length}_{S}(S / g(\mathfrak{m}) S) \leqslant \operatorname{length}_{S}(S / g(\mathfrak{q}) S)$

$$
+\operatorname{length}_{R}\left(\mathfrak{q} / \mathfrak{q}_{\alpha}\right) \cdot \operatorname{length}_{S}(S / g(\mathfrak{m}) S) .
$$

Now using equation (7) we obtain

$$
\operatorname{length}_{S}(S / g(\mathfrak{m}) S) \cdot \operatorname{length}_{R}(R / \mathfrak{q}) \leqslant \operatorname{length}_{S}(S / g(\mathfrak{q}) S) \text {. }
$$

Lemma 48. Let $(R, \mathfrak{m}, \varphi)$ be a local algebraic dynamical system, and let $\mathfrak{a}$ be a $\varphi$-stable ideal of $R$. Let $\bar{\varphi}$ be the endomorphism of $R / \mathfrak{a}$ induced by $\varphi$. Set $d:=\operatorname{dim} R$ and $\rho:=\operatorname{dim} R / \mathfrak{a}$ and let $q_{\varphi}:=\exp \left(h_{\mathrm{loc}}(\varphi) / d\right)$. Assume that length $\left(R / \varphi^{n}(\mathfrak{m}) R\right)=q_{\varphi}^{\text {nd }}$ for an integer $n \in \mathbb{N}$. Then

i) $\operatorname{length}\left(R / \varphi^{n t}(\mathfrak{m}) R\right)=q_{\varphi}^{\text {ntd }}$ for all $t \in \mathbb{N}$.

ii) If in addition $h_{\mathrm{loc}}(\bar{\varphi})=h_{\mathrm{loc}}(\varphi)$ and $\varphi$ is contracting, then $\mathfrak{a}=(0)$.

Proof: i) Fix $t \in \mathbb{N}$. As the sequence $\left\{\left(\log \operatorname{length}\left(\varphi^{n t}\right)\right) /(n t)\right\}$ by Theorem 1 converges to its infimum, we have

$$
h_{\mathrm{loc}}(\varphi) \leqslant \frac{1}{n t} \cdot \log \operatorname{length}\left(R / \varphi^{n t}(\mathfrak{m}) R\right), \quad \forall n \in \mathbb{N} .
$$

From this inequality we obtain $q_{\varphi}^{n t d} \leqslant \operatorname{length}\left(R / \varphi^{n t}(\mathfrak{m}) R\right)$. By Lemma 15a)

$$
\operatorname{length}\left(R / \varphi^{n t}(\mathfrak{m}) R\right) \leqslant\left(\operatorname{length}\left(R / \varphi^{n}(\mathfrak{m}) R\right)\right)^{t} .
$$

Using our hypothesis and previous inequalities we obtain

$$
q_{\varphi}^{n t d} \leqslant \operatorname{length}\left(R / \varphi^{n t}(\mathfrak{m}) R\right) \leqslant\left(\operatorname{length}\left(R / \varphi^{n}(\mathfrak{m}) R\right)\right)^{t}=q_{\varphi}^{n t d} .
$$

Hence, length $\left(R / \varphi^{n t}(\mathfrak{m}) R\right)=q_{\varphi}^{n t d}$ for all $t \in \mathbb{N}$.

ii) Similar to the previous part, we can write

$$
\begin{aligned}
q_{\bar{\varphi}}^{n t \rho} & \leqslant \operatorname{length}\left(\bar{\varphi}^{n t}\right)=\operatorname{length}\left(R /\left(\varphi^{n t}(\mathfrak{m}) R+\mathfrak{a}\right)\right) \\
& \leqslant \operatorname{length}\left(R / \varphi^{n t}(\mathfrak{m}) R\right)=q_{\varphi}^{n t d} .
\end{aligned}
$$


From our hypothesis in ii) we know $q_{\varphi}^{\rho}=q_{\varphi}^{d}$. Thus, from equation (8) we can conclude

(9) $\quad \operatorname{length}\left(R /\left(\varphi^{n t}(\mathfrak{m}) R+\mathfrak{a}\right)\right)=\operatorname{length}\left(R / \varphi^{n t}(\mathfrak{m}) R\right), \quad \forall t \in \mathbb{N}$.

The surjection $\left.R / \varphi^{n t}(\mathfrak{m}) R \rightarrow R /\left(\varphi^{n t}(\mathfrak{m}) R+\mathfrak{a}\right)\right) \rightarrow 0$ and equation (9) then show

$$
R /\left(\varphi^{n t}(\mathfrak{m}) R+\mathfrak{a}\right)=R / \varphi^{n t}(\mathfrak{m}) R, \quad \forall t \in \mathbb{N} .
$$

Hence,

$$
\mathfrak{a} \subset \bigcap_{t \in \mathbb{N}} \varphi^{n t}(\mathfrak{m}) R=(0),
$$

where the last equality follows from Lemma 4 because $\varphi$ is by assumption, contracting.

Theorem 2. Let $(R, \mathfrak{m}, \varphi)$ be a local algebraic dynamical system and let $d$ be the dimension of $R$. Let $h_{\mathrm{loc}}(\varphi)$ be the local entropy of this system and define $q_{\varphi}:=\exp \left(h_{\mathrm{loc}}(\varphi) / d\right)$. Consider the following conditions:

a) $R$ is regular.

b) $\varphi: R \rightarrow R$ is flat.

c) length $(R / \varphi(\mathfrak{m}) R)=q_{\varphi}^{d}$.

d) length $\left(R / \varphi^{n}(\mathfrak{m}) R\right)=q_{\varphi}^{\text {nd }}$ for an integer $n \in \mathbb{N}$.

Then $a) \Rightarrow b) \Rightarrow c$ ) $\Rightarrow d$ ). If in addition $\varphi$ is contracting, all these conditions are equivalent.

Proof: a) $\Rightarrow$ b) To say that $\varphi$ is of finite length means $\operatorname{dim} R / \varphi(\mathfrak{m}) R=0$. Hence, the following equation holds:

$$
\operatorname{dim} R=\operatorname{dim} R+\operatorname{dim} R / \varphi(\mathfrak{m}) R .
$$

Since $R$ is regular, the result follows from [35, Theorem 23.1].

b) $\Rightarrow$ c) Since $\varphi$ is flat, by Lemma $15 \mathrm{~b})$

$$
\operatorname{length}\left(R / \varphi^{n}(\mathfrak{m}) R\right)=(\operatorname{length}(R / \varphi(\mathfrak{m}) R))^{n}, \quad \forall n \in \mathbb{N} .
$$

Thus, by definition of local entropy

$$
\begin{aligned}
h_{\mathrm{loc}}(\varphi) & =\lim _{n \rightarrow \infty}(1 / n) \cdot \log \text { length }\left(R / \varphi^{n}(\mathfrak{m}) R\right) \\
& =\lim _{n \rightarrow \infty}(1 / n) \cdot \log (\operatorname{length}(R / \varphi(\mathfrak{m}) R))^{n} \\
& =\log \operatorname{length}(\varphi) .
\end{aligned}
$$

This means length $(R / \varphi(\mathfrak{m}) R)=q_{\varphi}^{d}$.

c) $\Rightarrow$ d) This is clear.

b) $\Rightarrow$ a) We rewrite Herzog's proof for the Frobenius endomorphism from [25, Satz 3.1], for an arbitrary endomorphism here. Bruns and 
Gubeladze have also used this proof in [12, Lemma 3]. We include it here for completeness. To show that $R$ is regular, it suffices to show all finitely generated $R$-modules have finite projective dimension. So let $M$ be a finitely generated $R$-module. Suppose $M$ were of infinite projective dimension. Consider a minimal (infinite) free resolution of $M$

$$
L_{\bullet} \rightarrow M \rightarrow 0 \text {. }
$$

Let $s:=\operatorname{depth}(\mathfrak{m}, R)$, and take an $R$-regular sequence of elements $\left\{x_{1}, \ldots\right.$, $\left.x_{s}\right\}$ in $\mathfrak{m}$. Write $\mathfrak{a}$ for the ideal generated by this regular sequence. (If $s=0$, take $\mathfrak{a}=(0)$.) Let $\Phi^{n}$ be the functor defined in Definition 17. For every $n \in \mathbb{N}$ we set

$$
C_{\bullet}^{n}:=\Phi^{n}\left(L_{\bullet}\right) \otimes_{R} R / \mathfrak{a} \quad \text { and } \quad B_{i}^{n}:=\operatorname{image}\left(C_{i+1}^{n} \rightarrow C_{i}^{n}\right) .
$$

Using properties of $\Phi^{n}$, see [40] or [25], it is easy to see that $C_{i}^{n} \cong L_{i} / \mathfrak{a} L_{i}$ and $B_{i}^{n} \subseteq \varphi^{n}(\mathfrak{m}) C_{i}^{n}$. Thus, for every $i$ the module $C_{i}^{n}$ is independent of $n$ and is nonzero, finitely generated and of depth zero. Applying Lemma 44 , let $\mu_{i_{0}}$ be $\operatorname{such}$ that $\operatorname{depth}\left(\mathfrak{m}, \mathfrak{m}^{\mu_{i_{0}}} C_{i}^{n}\right)>0$. Since $\varphi$ is contracting, using Lemma 4 if $n$ is large enough then $\varphi^{n}(\mathfrak{m}) R \subseteq \mathfrak{m}^{\mu_{i_{0}}}$, hence $B_{i}^{n} \subseteq \varphi^{n}(\mathfrak{m}) C_{i}^{n} \subseteq \mathfrak{m}^{\mu_{i}} C_{i}^{n}$. This shows that $\operatorname{depth}\left(\mathfrak{m}, B_{i}^{n}\right)>0$ for large $n$. On the other hand, since $\varphi$ is flat, $\Phi^{n}\left(L_{\bullet}\right)$ is exact. Thus, using properties of $\Phi^{n}$ again, we see that

$$
\Phi^{n}\left(L_{\bullet}\right) \rightarrow \Phi^{n}(M) \rightarrow 0
$$

is a minimal (infinite) free resolution of $\Phi^{n}(M)$. Hence

$$
\mathrm{H}_{i}\left(C_{\bullet}^{n}\right)=\operatorname{Tor}_{i}^{R}\left(\Phi^{n}(M), R / \mathfrak{a}\right)=0, \quad \text { for } \quad i>s .
$$

This shows that if $i>s$, then the sequences

$$
0 \rightarrow B_{i+1}^{n} \rightarrow C_{i+1}^{n} \rightarrow B_{i}^{n} \rightarrow 0
$$

are exact for all $n \in \mathbb{N}$. Take $i=s+1$ in sequence (10), for instance. By the above argument, if we take $n$ large enough, we will obtain $\operatorname{depth}\left(\mathfrak{m}, B_{s+1}^{n}\right)>0$ and $\operatorname{depth}\left(\mathfrak{m}, B_{s+2}^{n}\right)>0$, while $\operatorname{depth}\left(\mathfrak{m}, C_{s+2}^{n}\right)=0$. By Proposition 46 this is not possible. Hence, the projective dimension of $M$ must be finite.

d) $\Rightarrow$ b) We will use Nagata's Flatness Theorem to show that $\varphi^{n}$ is flat. We first need to show that $\varphi$ is injective. Clearly $\operatorname{ker} \varphi$ is $\varphi$-stable. Let $\bar{\varphi}$ be the local endomorphism induced by $\varphi$ on $R / \operatorname{ker} \varphi$. Then by Proposition $41, h_{\mathrm{loc}}(\varphi)=h_{\mathrm{loc}}(\bar{\varphi})$. By assumption, length $\left(R / \varphi^{n}(\mathfrak{m}) R\right)=$ $q_{\varphi}^{n d}$ for an integer $n \in \mathbb{N}$. From Lemma 48 it follows that $\operatorname{ker} \varphi=(0)$.

Now since $\varphi$ is contracting, using Lemma 4 we quickly see that the family $\left\{\varphi^{n t}(\mathfrak{m}) R\right\}_{t \in \mathbb{N}}$ defines the $\mathfrak{m}$-adic topology of $R$. By Lemma 47 
it suffices to verify equation (6) for this family of $\mathfrak{m}$-primary ideals. We need to show

$\operatorname{length}_{R}\left(R / \varphi^{n}\left(\varphi^{n t}(\mathfrak{m})\right) R\right)=\operatorname{length}_{R}\left(R / \varphi^{n t}(\mathfrak{m}) R\right) \cdot \operatorname{length}_{R}\left(R / \varphi^{n}(\mathfrak{m}) R\right)$.

Using Lemma 48, this equality holds, if and only if

$$
q_{\varphi}^{n(t+1) d}=q_{\varphi}^{n t d} \cdot q_{\varphi}^{n d}
$$

Since this equality holds trivially, by Nagata's Flatness Theorem $\varphi^{n}$ is flat. The implication $\mathrm{b}) \Rightarrow$ a) applied to $\varphi^{n}$ then tells us that $R$ is regular, and the implication a) $\Rightarrow$ b) shows that $\varphi$ is flat, as well.

Remark 49. There exist normal singularities $(X, P)$ such that $\mathcal{O}_{X, P}$ admits a finite contracting endomorphism, see [6, Sections 6.2-6.3] or [16, Sections 2.3-2.5]. In this case by Theorem 2 the endomorphism is not flat. This gives examples of finite maps between normal varieties which are not flat.

2.2. Generalized Hilbert-Kunz multiplicity. Following ideas of Kunz, Monsky in [37] defined the Hilbert-Kunz multiplicity for the Frobenius endomorphism of noetherian local rings of positive prime characteristic. He then showed that in this case, Hilbert-Kunz multiplicity always exists. Since then, it has become evident through works of various authors, that the Hilbert-Kunz multiplicity provides a reasonable measure of the singularity of the local ring. Here, inspired by Theorem 1d), we propose a characteristic-free interpretation of the definition of Hilbert-Kunz multiplicity associated with an endomorphism of finite length.

Definition 50 (Hilbert-Kunz multiplicity). Let $(R, \varphi)$ be a local algebraic dynamical system and set $d:=\operatorname{dim} R$. Let $q_{\varphi}:=\exp \left(h_{\mathrm{loc}}(\varphi, R) / d\right)$. The Hilbert-Kunz multiplicity of $R$ with respect to $\varphi$ is defined as

$$
e_{\mathrm{HK}}^{\varphi}(R):=\lim _{n \rightarrow \infty} \frac{\operatorname{length}\left(R / \varphi^{n}(\mathfrak{m}) R\right)}{q_{\varphi}^{n d}},
$$

provided that the limit exists.

Remark 51. We do not know whether the limit in equation (11) always exists or not. Nevertheless, the next corollary shows that in the case of a regular local ring the Hilbert-Kunz multiplicity is 1, as expected.

Corollary 52. Let $\varphi$ be an endomorphism of finite length of a regular local ring $R$. Then $e_{\mathrm{HK}}^{\varphi}(R)=1$.

Proof: This quickly follows from Theorem 2 and Lemma 15b). 
We end this section with a note. Not every homological property of the Frobenius endomorphism can be immediately extended to arbitrary endomorphisms. For example, in [40, Theorem 1.7, p. 58] Peskine and the third author showed that in positive prime characteristic, a finite free resolution of a module remains exact after applying the Frobenius functor (see Definition 17). This property may fail in general, for an arbitrary endomorphism, even in the simple case of a Koszul complex with one element. The image of a non-zerodivisor under an integral endomorphism could be a zerodivisor, as the next example shows.

Example 53. Consider the polynomial ring $k[x, y, z, w]$ over a field $k$. Let $\mathfrak{a}$ be the ideal $\left(x^{2}, x y, x z, z w\right)$ and let $A=k[x, y, z, w] / \mathfrak{a}$. Then

$$
\operatorname{Ass}(A)=\{(x, z),(x, w),(x, y, z)\} .
$$

Define an endomorphism $\varphi$ of $k[x, y, z, w]$ as $x \stackrel{\varphi}{\mapsto} x^{2} ; y \stackrel{\varphi}{\mapsto} y ; z \stackrel{\varphi}{\mapsto} w$; $w \stackrel{\varphi}{\mapsto} z$. Then $\mathfrak{a}$ is $\varphi$-stable. Let $\bar{\varphi}$ be the endomorphism of $A$ induced by $\varphi$. The $A$-module $\bar{\varphi}_{*} A$ is finitely generated. In fact, it is generated by 1 and $x$ as an $A$-module. Now, $y+w$ is not a zerodivisor in $A$ because it does not belong to any prime ideal in $\operatorname{Ass}(A)$. But $\bar{\varphi}(y+w)=y+z$ is a zerodivisor in $A$; it is killed by $x$, for example. On the other hand, $y+z$ is a zerodivisor but is mapped to $y+w$, a non-zerodivisor.

Nonetheless, in the previous example $\bar{\varphi}^{2}$ sends any $A$-regular sequence to an $A$-regular sequence. This motivates the following

Question 54. Let $(R, \varphi)$ be a local algebraic dynamical system. Does there exist a positive integer $n$ such that $\varphi^{n}$ will send any $R$-regular sequence to an $R$-regular sequence?

\subsection{Endomorphisms of complete equicharacteristic local rings.} In this section we prove Theorem 3, which is inspired by results of Fakhruddin [15, Corollary 2.2], and Bhatnagar and the third author [5, Theorem 2.1] on extending a polarized self-morphism of a projective variety over an infinite field to an ambient projective space. Recently in [41] Poonen gave a proof for the main result of [5] over finite fields.

Consider a self-morphism $\varphi$ of a projective variety $X$ over an infinite field $k$ and let $\mathcal{L}$ be an ample line bundle on $X$ with $\varphi^{*}(\mathcal{L}) \cong \mathcal{L}^{\otimes q}$ for an integer $q \geqslant 1$. In [15] Fakhruddin showed that there exists an embedding $\imath$ of $X$ in $\mathbb{P}_{k}^{N}$ given by an appropriate tensor power of $\mathcal{L}$ and a self-morphism $\psi$ of $\mathbb{P}_{k}^{N}$ such that $\psi \circ \imath=\imath \circ \varphi$. In [5] Bhatnagar and the third author relaxed some of Fakhruddin's hypotheses and showed that (assuming $\mathcal{L}$ is very ample) one can keep the same embedding of $X$ given 
by $\mathcal{L}$ and instead extend an appropriate iteration of $\varphi$ to the ambient projective space.

Our Theorem 3 is an analogous result about lifting of endomorphisms of finite length of complete noetherian local rings of equal characteristic. In this local version of Fakhruddin's result we do not assume our fields to be infinite. We will begin with a few preparatory results that we will need in the proof of Theorem 3.

Definition 55 ([44, p. 159]). In a noetherian local ring $R$ of dimension $d$ and of embedding dimension $\delta$, a system of parameters $\left\{x_{1}, \ldots, x_{d}\right\}$ is called a strong system of parameters if it is part of a minimal set of generators $\left\{x_{1}, \ldots, x_{d}, \ldots, x_{\delta}\right\}$ of the maximal ideal.

Lemma 56. A noetherian local ring $(R, \mathfrak{m})$ has strong systems of parameters.

Proof: The proof is by induction on $\operatorname{dim} R$. If $\operatorname{dim} R=0$ then the statement is vacuous, since every system of parameters is empty. So assume $\operatorname{dim} R>0$ and using the Prime Avoidance Lemma [34, p. 2], pick an element $x \in \mathfrak{m}$ that is neither in any minimal prime ideal of $R$, nor in $\mathfrak{m}^{2}$. Apply the induction hypothesis to $R /\langle x\rangle$.

Lemma 57. Let $(R, \mathfrak{m})$ be a complete local ring of equal characteristic and assume that $A$ is a homomorphic image $\pi: R \rightarrow A$ of $R$. If $K$ is a subfield of $A$, then there is a subfield $L$ of $R$ such that $\left.\pi\right|_{L}: L \rightarrow K$ is an isomorphism.

Proof: Let $B=\pi^{-1}(K)$. Then $B$ is a local subring of $R$ with maximal ideal $\mathfrak{q}=\pi^{-1}(0)$. Note that $\mathfrak{q}=\operatorname{ker} \pi$ as subsets of $R$. Since $B / \mathfrak{q} \cong K$, $B$ is also of equal characteristic. In general $B$ need not be noetherian. We claim that $B \subseteq R$ is a closed subset in the m-adic topology of $R$. To see this, let $\mathfrak{n}$ be the maximal ideal of $A$ and note that the topology induced from the n-adic topology of $A$ on any subfield of $A$ is the discrete topology. Therefore, any subfield of $A$ is complete with respect to the topology induced from $A$, and hence is closed in $A$. Since $\pi$ is a continuous map and $B=\pi^{-1}(K)$, the claim follows. In particular, $B$ is complete with respect to the topology induced from the $\mathfrak{m}$-adic topology of $R$.

Denote the $\mathfrak{q}$-adic completion of $B$ by $\hat{B}$. Since $B$ is a local subring of $R$ and $R$ is complete, we obtain a map $\hat{i}: \widehat{B} \rightarrow R$, where $i: B \hookrightarrow R$ is the inclusion homomorphism. Furthermore, since $B$ is complete with respect to the topology induced from the $\mathfrak{m}$-adic topology of $R$, we see that $\hat{i}(\widehat{B})=B$. Let $L^{\prime}$ be a coefficient field of $\widehat{B}$. (For the existence of 
coefficient fields in complete local rings that are not necessarily noetherian, see [38, Theorem 31.1], or [35, Theorem 28.3] or [21, Corollary 2].) Let $L:=\hat{i}\left(L^{\prime}\right)$. Then $L$ is subfield of $B$ that is isomorphic to $L^{\prime}$. Furthermore, the following diagram is commutative, and shows that $\left.\pi\right|_{L}: L \rightarrow K$ is an isomorphism.

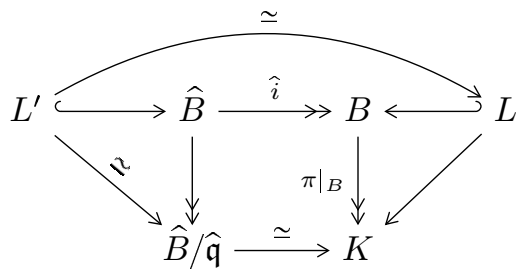

Theorem 3. Let $(A, \mathfrak{n}, \varphi)$ be a local algebraic dynamical system and assume that $A$ is a homomorphic image $\pi: R \rightarrow A$ of an equicharacteristic complete regular local ring $(R, \mathfrak{m})$. Then $\varphi$ can be lifted (non uniquely) to an endomorphism of finite length $\psi$ of $R$ such that $\pi \circ \psi=\varphi \circ \pi$. Thus, $\pi:(R, \psi) \rightarrow(A, \varphi)$ becomes a morphism of local algebraic dynamical systems.

Proof: Let $K$ be an arbitrary coefficient field of $R$. Then $\varphi(\pi(K))$ is a subfield of $A$, and can be lifted to a subfield $L$ of $R$, by Lemma 57 , in such a way that $\left.\pi\right|_{L}: L \rightarrow \varphi(\pi(K))$ is an isomorphism. We will use $L$ at the end of our proof to construct an endomorphism $\psi$ of $R$. Let $d=\operatorname{dim} A$ and let $\delta$ be the embedding dimension of $A$. By Lemma 56 we can choose a strong system of parameters $\left\{x_{1}, \ldots, x_{d}\right\}$ of $A$ which is part of a minimal set of generators $\left\{x_{1}, \ldots, x_{d}, \ldots, x_{\delta}\right\}$ of $\mathfrak{n}$. Choose elements $X_{1}, \ldots, X_{\delta}$ in $\mathfrak{m}$ in such a way that $\pi\left(X_{i}\right)=x_{i}$ for each $i$. We claim that since the images of $x_{1}, \ldots, x_{\delta}$ in $\mathfrak{n} / \mathfrak{n}^{2}$ are linearly independent over $A / \mathfrak{n}$, the images $\bar{X}_{1}, \ldots, \bar{X}_{\delta}$ of $X_{1}, \ldots, X_{\delta}$ in $\mathfrak{m} / \mathfrak{m}^{2}$ are also linearly independent over $R / \mathfrak{m}$. If not, there will be a dependence relation $\alpha_{1} \bar{X}_{1}+\cdots+\alpha_{\delta} \bar{X}_{\delta}=0$ with $\alpha_{i} \in R / \mathfrak{m}$ not all zero. This means if we choose $a_{i} \in R$ such that they map to $\alpha_{i}$ in $R / \mathfrak{m}$ for $1 \leqslant i \leqslant \delta$, then

$$
a_{1} X_{1}+\cdots+a_{\delta} X_{\delta} \in \mathfrak{m}^{2} .
$$

If we apply $\pi$ to this relation, we obtain $\pi\left(a_{1}\right) x_{1}+\cdots+\pi\left(a_{\delta}\right) x_{\delta} \in \mathfrak{n}^{2}$. But then the image in $\mathfrak{n} / \mathfrak{n}^{2}$ would provide a nontrivial dependence relation

$$
\pi\left(a_{1}\right) \bar{x}_{1}+\cdots+\pi\left(a_{\delta}\right) \bar{x}_{\delta}=0,
$$


contradicting the linear independence of $\bar{x}_{1}, \ldots, \bar{x}_{\delta}$ in $\mathfrak{n} / \mathfrak{n}^{2}$ over $A / \mathfrak{n}$. Our claim follows. Hence, we can extend $\left\{\bar{X}_{1}, \ldots, \bar{X}_{\delta}\right\}$ to a basis $\left\{\bar{X}_{1}, \ldots\right.$, $\left.\bar{X}_{\delta}, \ldots, \overline{X_{n}}\right\}$ of $\mathfrak{m} / \mathfrak{m}^{2}$ over $R / \mathfrak{m}$, where $n=\operatorname{dim} R$. If we choose elements $X_{i} \in \mathfrak{m}$ such that they map to $\bar{X}_{i}$ in $\mathfrak{m} / \mathfrak{m}^{2}$ for $\delta+1 \leqslant i \leqslant n$, then by Nakayama's Lemma $\left\{X_{1}, \ldots, X_{n}\right\}$ is a minimal set of generators of $\mathfrak{m}$. Furthermore, it follows from the Cohen Structure Theorem that $R=K \llbracket X_{1}, \ldots, X_{n} \rrbracket$.

Now consider elements $\varphi\left(\pi\left(X_{i}\right)\right)$ in $A$ and for $1 \leqslant i \leqslant d$ choose $f_{i} \in \mathfrak{m}$ such that $\pi\left(f_{i}\right)=\varphi\left(\pi\left(X_{i}\right)\right)$. We claim that the ideal $\left\langle f_{1}, \ldots, f_{d}\right\rangle$ of $R$ has height $d$. First, by Krull's Theorem ht $\left\langle f_{1}, \ldots, f_{d}\right\rangle \leqslant d$. For the inequality in the other direction, note that the ideal $\mathfrak{b}:=\left\langle\varphi\left(\pi\left(X_{1}\right)\right), \ldots\right.$, $\left.\varphi\left(\pi\left(X_{d}\right)\right)\right\rangle$ is $\mathfrak{n}$-primary. Hence, $\pi^{-1}(\mathfrak{b})=\left\langle f_{1}, \ldots, f_{d}\right\rangle+\operatorname{ker} \pi$ is an $\mathfrak{m}$-primary ideal in $R$. Since $R$ is regular, by Serre's Intersection Theorem [45, Chapter V, Theorem 1]

$$
\operatorname{dim}(R / \operatorname{ker} \pi)+\operatorname{dim}\left(R /\left\langle f_{1}, \ldots, f_{d}\right\rangle\right) \leqslant \operatorname{dim} R,
$$

or, $d+\left(\operatorname{dim} R /\left\langle f_{1}, \ldots, f_{d}\right\rangle\right) \leqslant n$. But $\operatorname{dim}\left(R /\left\langle f_{1}, \ldots, f_{d}\right\rangle\right)=n-$ ht $\left\langle f_{1}, \ldots, f_{d}\right\rangle$ as $R$ is regular. We obtain ht $\left\langle f_{1}, \ldots, f_{d}\right\rangle \geqslant d$ and our claim follows.

Next, we will choose elements $f_{d+1}, \ldots, f_{n} \in \mathfrak{m}$ inductively, making sure at each step that $\pi\left(f_{t}\right)=\varphi\left(\pi\left(X_{t}\right)\right)$ and that $\operatorname{dim} R /\left\langle f_{1}, \ldots, f_{t}\right\rangle=$ $n-t$. Assume $d \leqslant t<n$ and that $f_{1}, \ldots, f_{t}$ have been chosen with desired properties. To choose $f_{t+1}$ we use the coset version of the Prime Avoidance Lemma due to E. Davis (see [27, Theorem 124] or [35, Exercise 16.8]), that can be stated as follows: let $I$ be an ideal of a commutative ring $R$ and $x \in R$ be an element. Let $\mathfrak{p}_{1}, \ldots, \mathfrak{p}_{s}$ be prime ideals of $R$ none of which contain $I$. Then

$$
x+I \nsubseteq \bigcup_{i=1}^{s} \mathfrak{p}_{i} .
$$

Choose an element $u \in \mathfrak{m}$ such that $\pi(u)=\varphi\left(\pi\left(X_{t+1}\right)\right)$. If

$$
\operatorname{dim} R /\left\langle f_{1}, \ldots, f_{t}, u\right\rangle=n-t-1,
$$

then set $f_{t+1}=u$. If not, let $\left\{\mathfrak{p}_{1}, \ldots, \mathfrak{p}_{s}\right\}$ be the set of minimal associated prime ideals of $R /\left\langle f_{1}, \ldots, f_{t}\right\rangle$ that satisfy

$$
\operatorname{dim} R / \mathfrak{p}_{i}=\operatorname{dim} R /\left\langle f_{1}, \ldots, f_{t}\right\rangle .
$$

Since $\left\langle f_{1}, \ldots, f_{t}\right\rangle+$ ker $\pi$ is an $\mathfrak{m}$-primary ideal in $R$, none of these $\mathfrak{p}_{i}$ 's can contain ker $\pi$. Therefore by the coset version of the Prime Avoidance Lemma there exists an element $a \in \operatorname{ker} \pi$ such that

$$
u+a \notin \bigcup_{i=1}^{s} \mathfrak{p}_{i} .
$$


Setting $f_{t+1}=u+a$ we see that $\operatorname{dim} R /\left\langle f_{1}, \ldots, f_{t+1}\right\rangle=n-t-1$ and $\pi\left(f_{t+1}\right)=\varphi\left(\pi\left(X_{t+1}\right)\right)$, as desired.

After choosing $\left\{f_{1}, \ldots, f_{n}\right\}$ as described, we define an endomorphism $\psi$ of $R=K \llbracket X_{1}, \ldots, X_{n} \rrbracket$ as follows. For each $1 \leqslant i \leqslant n$, we define $\psi\left(X_{i}\right)$ to be $f_{i}$ and for every element $\alpha$ of $K$ we define $\psi(\alpha)$ to be $\left(\left.\pi\right|_{L}\right)^{-1}(\varphi(\pi(\alpha)))$. Then we extend the definition of $\psi$ to all elements of $R$ by continuity. Since $\psi(\mathfrak{m}) R=\left\langle f_{1}, \ldots, f_{n}\right\rangle$ is $\mathfrak{m}$-primary by construction of the $f_{i}$ 's, $\psi$ is of finite length. Moreover, it is clear from the construction that $\varphi \circ \pi=\pi \circ \psi$, that is, $\pi:(R, \psi) \rightarrow(A, \varphi)$ is a morphism of local algebraic dynamical systems.

Corollary 58. If $\varphi$ in Theorem 3 is finite, then so is $\psi$.

Proof: This follows from [13, Theorem 8]: a local homomorphism $f: S \rightarrow$ $T$ of complete noetherian local rings is finite if and only if $f$ is of finite length, and $\left[f_{*} k_{T}: k_{S}\right]$ is a finite (algebraic) field extension, where $k_{S}$ and $k_{T}$ are residue fields of $S$ and $T$.

Question 59. Is it possible in Theorem 3 to take $\psi$ to satisfy $v_{h}(\psi)=$ $v_{h}(\varphi), w_{h}(\psi)=w_{h}(\varphi)$ and $(\operatorname{dim} A) \cdot h_{\mathrm{loc}}(\psi)=(\operatorname{dim} R) \cdot h_{\mathrm{loc}}(\varphi) ?$

\section{Acknowledgements}

The authors would like to thank the referees for their detailed and insightful suggestions.

\section{References}

[1] R. L. Adler, A. G. Konheim, And M. H. McAndrew, Topological entropy, Trans. Amer. Math. Soc. 114 (1965), 309-319. DOI: 10.1090/S0002-9947-1965-0175106-9.

[2] L. L. Avramov, M. Hochster, S. B. Iyengar, and Y. Yao, Homological invariants of modules over contracting endomorphisms, Math. Ann. 353(2) (2012), 275-291. DOI: 10.1007/s00208-0110682-z.

[3] L. L. Avramov, S. Iyengar, And C. Miller, Homology over local homomorphisms, Amer. J. Math. 128(1) (2006), 23-90. DOI: 10.1353/ajm. 2006.0001.

[4] M. P. Bellon and C.-M. Viallet, Algebraic entropy, Comm. Math. Phys. 204(2) (1999), 425-437. DOI: 10.1007/s002200050652.

[5] A. Bhatnagar And L. SzPiro, Very ample polarized self maps extend to projective space, J. Algebra 351 (2012), 251-253. DOI: 10.1016/j · jalgebra.2011.11.010. 
[6] S. Boucksom, T. De Fernex, and C. Favre, The volume of an isolated singularity, Duke Math. J. 161(8) (2012), 1455-1520. DOI: 10.1215/00127094-1593317.

[7] N. Bourbaki, "Algebra. I. Chapters 1-3", Translated from the French, Reprint of the 1974 edition, Elements of Mathematics (Berlin), Springer-Verlag, Berlin, 1989.

[8] N. Bourbaki, "Commutative algebra. Chapters 1-7", Translated from the French, Reprint of the 1972 edition, Elements of Mathematics (Berlin), Springer-Verlag, Berlin, 1989.

[9] N. Bourbaki, "Éléments de mathématique. Algèbre commutative. Chapitre 10", Reprint of the 1998 original, Springer-Verlag, Berlin, 2007.

[10] H. Brolin, Invariant sets under iteration of rational functions, Ark. Mat. 6 (1965), 103-144. DOI: 10.1007/BF02591353.

[11] A. Broustet And A. Hoering, Singularities of varieties admitting an endomorphism, Preprint (2012), arXiv:1210.6254.

[12] W. Bruns and J. Gubeladze, A regularity criterion for semigroup rings, Georgian Math. J. 6(3) (1999), 259-262. DOI: 10.1023/ A : 1022183113841.

[13] I. S. Cohen, On the structure and ideal theory of complete local rings, Trans. Amer. Math. Soc. 59 (1946), 54-106. DOI: 10.1090/S0002-9947-1946-0016094-3.

[14] T.-C. Dinh And N. Sibony, Equidistribution towards the Green current for holomorphic maps, Ann. Sci. Éc. Norm. Supér (4) 41(2) (2008), 307-336.

[15] N. FAKHRUdDin, Questions on self maps of algebraic varieties, $J$. Ramanujan Math. Soc. 18(2) (2003), 109-122.

[16] C. FAVRe, Holomorphic self-maps of singular rational surfaces, Publ. Mat. 54(2) (2010), 389-432. DOI: 10.5565/PUBLMAT_54210_06.

[17] C. Favre and M. Jonsson, Brolin's theorem for curves in two complex dimensions, Ann. Inst. Fourier (Grenoble) 53(5) (2003), 1461-1501. DOI: 10.5802/aif.1985.

[18] C. Favre and M. Jonsson, "The valuative tree", Lecture Notes in Mathematics 1853, Springer-Verlag, Berlin, 2004. DOI: 10.1007/ b100262.

[19] C. Favre and M. Jonsson, Eigenvaluations, Ann. Sci. École Norm. Sup. (4) 40(2) (2007), 309-349. DOI: 10.1016/j.ansens. 2007.01 .002 .

[20] J. E. Fornæss and N. Sibony, Complex dynamics in higher dimension. II, in: "Modern methods in complex analysis" (Princeton, 
NJ, 1992), Ann. of Math. Stud. 137, Princeton Univ. Press, Princeton, NJ, 1995, pp. 135-182.

[21] A. Geddes, On coefficient fields, Proc. Glasgow Math. Assoc. 4 (1958), 42-48.

[22] W. Gignac and M. Ruggiero, Growth of attraction rates for iterates of a superattracting germ in dimension two, Preprint (2012), arXiv: 1209.3450v1.

[23] M. Gromov, On the entropy of holomorphic maps, Enseign. Math. (2) 49(3-4) (2003), 217-235.

[24] B. Hasselblatt and J. Propp, Degree-growth of monomial maps, Ergodic Theory Dynam. Systems 27(5) (2007), 1375-1397. DOI : $10.1017 /$ S0143385707000168.

[25] J. Herzog, Ringe der Charakteristik $p$ und Frobeniusfunktoren, Math. Z. 140 (1974), 67-78. DOI: 10.1007/BF01218647.

[26] C. Huneke and R. Wiegand, Correction to: "Tensor products of modules and the rigidity of Tor" [Math. Ann. 299(3) (1994), 449-476]. Math. Ann. 338(2) (2007), 291-293. DOI: 10.1007/ s00208-006-0076-9.

[27] I. Kaplansky, "Commutative rings", Revised edition, The University of Chicago Press, Chicago, Ill.-London, 1974.

[28] A. N. Kolmogorov, A new metric invariant of transient dynamical systems and automorphisms in Lebesgue spaces, (Russian), Dokl. Akad. Nauk SSSR (N.S.) 119 (1958), 861-864.

[29] E. Kunz, Characterizations of regular local rings for characteristic p, Amer. J. Math. 91 (1969), 772-784.

[30] E. Kunz, On Noetherian rings of characteristic p, Amer. J. Math. 98(4) (1976), 999-1013.

[31] D. LAZARD, Algèbre linéaire sur $K\left[X_{1}, \ldots, X_{n}\right]$, et élimination, Bull. Soc. Math. France 105(2) (1977), 165-190.

[32] M. Ju. LJUbich, Entropy properties of rational endomorphisms of the Riemann sphere, Ergodic Theory Dynam. Systems 3(3) (1983), 351-385. DOI : $10.1017 /$ S0143385700002030.

[33] J. Llibre And R. Saghin, Results and open questions on some invariants measuring the dynamical complexity of a map, Fund. Math. 206 (2009), 307-327. DOI: 10.1007/10.4064/fm206-0-19.

[34] H. Matsumura, "Commutative algebra", Second edition, Mathematics Lecture Note Series 56, Benjamin/Cummings Publishing Co., Inc., Reading, Mass., 1980.

[35] H. Matsumura, "Commutative ring theory", Translated from the Japanese by M. Reid, Cambridge Studies in Advanced Mathematics 8, Cambridge University Press, Cambridge, 1986. 
[36] M. Misiurewicz And F. Przytycki, Topological entropy and degree of smooth mappings, Bull. Acad. Polon. Sci. Sér. Sci. Math. Astronom. Phys. 25(6) (1977), 573-574.

[37] P. Monsky, The Hilbert-Kunz function, Math. Ann. 263(1) (1983), 43-49. DOI : 10.1007/BF01457082.

[38] M. Nagata, "Local rings", Corrected reprint, Robert E. Krieger Publishing Co., Huntington, N.Y., 1975.

[39] M. R. PARRA, The Jacobian cocycle and equidistribution towards the Green current, Preprint (2011), arXiv:1103.4633v1.

[40] C. Peskine and L. SzPiro, Dimension projective finie et cohomologie locale. Applications à la démonstration de conjectures de M. Auslander, H. Bass et A. Grothendieck, Inst. Hautes Études Sci. Publ. Math. 42 (1973), 47-119.

[41] B. Poonen, Extending self-maps to projective space, Preprint (2012).

[42] A. G. Rodicio, On a result of Avramov, Manuscripta Math. 62(2) (1988), 181-185. DOI: $10.1007 / \mathrm{BF} 01278977$.

[43] P. Samuel, Some asymptotic properties of powers of ideals, Ann. of Math. (2) 56 (1952), 11-21.

[44] H. Schoutens, "The use of ultraproducts in commutative algebra", Lecture Notes in Mathematics 1999, Springer-Verlag, Berlin, 2010. DOI : $10.1007 / 978-3-642-13368-8$.

[45] J.-P. Serre, "Algèbre locale. Multiplicités", Cours au Collège de France, 1957-1958, rédigé par Pierre Gabriel, Seconde édition, Lecture Notes in Mathematics 11, Springer-Verlag, Berlin-New York, 1965.

[46] J. H. Silverman, Dynamical degrees, arithmetic degrees, and canonical heights for dominant rational self-maps of projective space, Preprint (2012), arXiv:1111.5664.

[47] JA. SinaĬ, On the concept of entropy for a dynamic system, (Russian), Dokl. Akad. Nauk SSSR 124 (1959), 768-771.

[48] L. SzPiro, E. Ullmo, and S. Zhang, Équirépartition des petits points, Invent. Math. 127(2) (1997), 337-347. DOI: 10.1007 /s002220050123.

[49] J. TAFLin, Equidistribution speed towards the Green current for endomorphisms of $\mathbb{P}^{k}$, Adv. Math. 227(5) (2011), 2059-2081. DOI: 10.1007/10.1016/j.aim.2011.04.010.

[50] P. Walters, "An introduction to ergodic theory", Graduate Texts in Mathematics 79, Springer-Verlag, New York-Berlin, 1982. 
[51] L.-S. Young, Entropy in dynamical systems, in: "Entropy", Princeton Ser. Appl. Math., Princeton Univ. Press, Princeton, NJ, 2003, pp. 313-327.

Mahdi Majidi-Zolbanin:

Department of Mathematics

LaGuardia Community College of the City University of New York

31-10 Thomson Avenue

Long Island City, NY 11101

USA

E-mail address: mmajidi-zolbanin@lagcc.cuny.edu

Nikita Miasnikov and Lucien Szpiro:

Department of Mathematics

The Graduate Center of the City University of New York

365 Fifth Avenue

New York, NY 10016

USA

E-mail address: n5k5t5@gmail.com

E-mail address: lszpiro@gc.cuny.edu

Primera versió rebuda el 30 de juliol de 2012, darrera versió rebuda el 17 de desembre de 2012 . 